\title{
T-Abso and T-Abso Quasi Primary Fuzzy Submodules
}

\author{
Wafaa H. Hanoon \\ Department of Computer Science, College of Education for Girls, University of Kufa, Iraq. \\ wafaah.hannon@uokufa.edu.iq \\ Hatem Y. Khalaf \\ Department of Mathematics, College of Education for Pure Science Ibn- Haitham \\ University of Baghdad, Baghdad, Iraq. \\ dr.hatamyahya@yahoo.com
}

Article history: Received 4 November 2018, Accepted 24 November 2018, Publish January 2019

\begin{abstract}
Let $M$ be a unitary $\mathrm{R}$-module and $\mathrm{R}$ is a commutative ring with identity. Our aim in this paper to study the concepts T-ABSO fuzzy ideals, T-ABSO fuzzy submodules and T-ABSO quasi primary fuzzy submodules, also we discuss these concepts in the class of multiplication fuzzy modules and relationships between these concepts. Many new basic properties and characterizations on these concepts are given.
\end{abstract}

Keywords: T-ABSO fuzzy ideal, T-ABSO fuzzy submodule, Quasi- prime fuzzy submodule, T-ABSO primary fuzzy submodule, T-ABSO quasi primary fuzzy submodule, Multiplication fuzzy module.

\section{Introduction}

In this paper all ring is commutative with identity and all modules are unitary. Deniz S. et al in [1] presented the concept of 2-absorbing fuzzy ideal which is a generalization of prime fuzzy ideal. Prime submodule which play an important turn in the module theory over a commutative ring. A prime submodule $N$ of an R-module $\dot{M}, N \neq \dot{M}$, with property $a \in R, x \in \dot{M}$, $a x \in N$ implies that $\in N$ or $a \in(N: M)$ [2]. This concept was generalized to concept of prime fuzzy submodule which was presented by Rabi [3]. In 1999, Abdul-Razakm, presented and studied quasi-prime submodule let $N<\dot{M}, N$ be called a quasi-prime if for $a, b \in R, m \in M$, abmeN, implies either $a m \in N$ or bmeN [4]. In 2001, Hatam generalized it to fuzzy quasi-prime submodules [5]. Darani, et al in [6] presented the definition of 2-absorbing submodule. Let $\mathrm{N}<\dot{M}, N$ be called 2-absorbing submodule of $\dot{M}$ if whenever $\mathrm{r}, b \in R, x \in \dot{M}$ and $r b x \in N$, then $r x \in N$ or $b x \in N$ or $\mathrm{rb} \in(N: M)$. Hatam and wafaa expanded this concept that is: if $X$ be a fuzzy module of an Rmodule $\dot{M}$. A proper fuzzy submodule $A$ of $X$ is called T-ABSO fuzzy submodule if whenever $a_{s}, b_{l}$ be fuzzy singletons of $\mathrm{R}$, and $x_{v} \subseteq X, \forall s, l, v \in L$, such that $a_{s} b_{l} x_{v} \subseteq A$, then either $a_{s} b_{l} \subseteq\left(A:_{R} X\right)$ or $a_{s} x_{v} \subseteq A$ or $b_{l} x_{v} \subseteq A$ [7]. McCasland and Moore presented the concept of $\dot{M}$-radical of $N$ such: Let $N$ be a proper module of a nonzero R-module $\dot{M}$, then the $\dot{M}$-radical of $N$, denoted by $M-\operatorname{rad} N$ is defined to be the intersection of all prime module including $N$, see [8]. Mostafanasab et al, were presented the connotation of 2-absorbing primary submodule. So, A proper submodule $N$ of an R-module $\dot{M}$ is called 2-absorbing primary submodule of $\dot{M}$ if whenever $a, b \in \mathrm{R}$ and $m \in \dot{M}$ and $a b m \in N$, then $a m \in M^{\prime}-\operatorname{rad} N$ or $b m \in \dot{M}-\operatorname{rad} N$ or $a b \in\left(N:_{R} \dot{\mathrm{M}}\right)$, 
[9]. Rabi and Hassan in 2008 were presented the concept of quasi primary fuzzy submodule. A proper fuzzy submodule $A$ of fuzzy module $X$ is said to be quasi primary fuzzy submodule if $(A: B)$ is a primary fuzzy ideal of $\mathrm{R}$ for each fuzzy submodule $B$ of $X$ such that $A \subset B$ [10]. Suat $\mathrm{K}$. et al, studied and presented the connotation of 2-absorbing quasi primary submodule, i.e., A proper submodule $N$ of $\dot{M}$ is said to be 2-absorbing quasi primary submodule if the condition $a b q \in N$ implies either $a b \in \sqrt{N:_{R} \dot{M}}$ or $a q \in \dot{M}-\operatorname{rad}(N)$ or $b q \in \dot{M}-\operatorname{rad}(N)$ for every $a$, $b \in \mathrm{R}$ and $q \in M$ [11]. This paper is composed of two sections.

In section (1) we present the definition of T-ABSO fuzzy ideals and we give some characterizations of this definition for ideals. Also many properties and outcomes of this concept are given. In section (2) we present the definition of T-ABSO fuzzy submodules, many basic properties and outcomes are studied. In section (3) we present the concept of T-ABSO quasi primary fuzzy submodules and we study the relationships this concept with among TABSO fuzzy submodules and T-ABSO primary fuzzy submodules. Several important results have been demonstrated. Note that we denote to fuzzy module, submodule.

\section{T-ABSO F. Ideals}

In this section, we introduce the concepts of T-ABSO and T-ABSO primary ideals. Some concepts and propositions which are needed in the next section.

\section{Definition 1. [1]}

Let $\hat{\mathrm{H}}$ be a non-constant $\mathrm{F}$. ideal of $\mathrm{R}$. Then $\hat{\mathrm{H}}$ is called T-ABSO F. ideal if for any F. points $a_{s}, b_{l}, r_{k}$ of $\mathrm{R}, a_{s} b_{l} r_{k} \in \hat{\mathrm{H}}$ implies that either $a_{s} b_{l} \in \hat{\mathrm{H}}$ or $a_{s} r_{k} \in \hat{\mathrm{H}}$ or $b_{l} r_{k} \in \hat{\mathrm{H}}$.

The following proposition characterize T-ABSO F. ideal in terms of its level ideal.

\section{Lemma 2. [1]}

Let $A$ be F. ideal of R. If $A$ is T-ABSO F. ideal, then $A_{v}$ is T-ABSO ideal of $\mathrm{R}, \forall v \in \mathrm{L}$,

Recall that Let $\hat{\mathrm{H}}$ be any F. ideal of R. Then the radical F. of $\hat{\mathrm{H}}$, denoted by $\sqrt{\hat{\mathrm{H}}}$, is defined by: $\sqrt{\hat{\mathrm{H}}}=\bigcap\{\mathrm{U}: \mathrm{U}$ is a prime F. ideal of R containing $\hat{\mathrm{H}}\}[$ [12].

Now, we give these propositions which are used in the next section.

\section{Proposition 3.}

Suppose that $\mathrm{R}$ be a ring and $\hat{\mathrm{H}}$ is T-ABSO F. ideal of R. Then $\sqrt{\hat{\mathrm{H}}}$ is T-ABSO F. ideal of $\mathrm{R}$ and $a_{v}^{2} \subseteq \hat{\mathrm{H}}$ for each F. singleton $a_{v} \subseteq \sqrt{\hat{\mathrm{H}}}, \forall v \in \mathrm{L}$.

Proof. Let $\hat{\mathrm{H}}$ be T-ABSO F. ideal and $a_{v} \subseteq \sqrt{\hat{\mathrm{H}}}$, hence $a \in \sqrt{\hat{\mathrm{H}}_{v}}$. Then $a^{2} \in \hat{\mathrm{H}}_{v}$. So that $\hat{\mathrm{H}}\left(a^{2}\right) \geq v$. Thus $\left(a_{v}\right)^{2} \subseteq \hat{\mathrm{H}}$. Since $\left(a_{v}\right)^{2}=a_{v}^{2}$, so that $a_{v}^{2} \subseteq \hat{\mathrm{H}}$. Now, let $a_{s}, b_{l}, r_{k}$ be F. singletons of $\mathrm{R}$ such that $a_{s} b_{l} r_{k} \subseteq \sqrt{\hat{\mathrm{H}}}$. Then $\left(a_{s} b_{l}, r_{k}\right)^{2}=a_{s}^{2} b_{l}^{2} r_{k}^{2} \subseteq \hat{\mathrm{H}}$. Since $\hat{\mathrm{H}}$ is TABSO F. ideal, then either $a_{s}^{2} b_{l}^{2} \subseteq \hat{\mathrm{H}}$ or $a_{s}^{2} r_{k}^{2} \subseteq \hat{\mathrm{H}}$ or $b_{l}^{2} r_{k}^{2} \subseteq \hat{\mathrm{H}}$, since $\left(a_{s} b_{l}\right)^{2}=a_{s}^{2} b_{l}^{2}$, $\left(a_{s} r_{k}\right)^{2}=a_{s}^{2} r_{k}^{2},\left(b_{l} r_{k}\right)^{2}=b_{l}^{2} r_{k}^{2}$ hence either $\left(a_{s} b_{l}\right)^{2} \subseteq \hat{\mathrm{H}}$ or $\left(a_{s} r_{k}\right)^{2} \subseteq \hat{\mathrm{H}}$ or $\left(b_{l} r_{k}\right)^{2} \subseteq$ $\hat{\mathrm{H}}$. So that either $a_{s} b_{l} \subseteq \sqrt{\hat{\mathrm{H}}}$ or $a_{s} r_{k} \subseteq \sqrt{\hat{\mathrm{H}}}$ or $b_{l} r_{k} \subseteq \sqrt{\hat{\mathrm{H}}}$. Thus $\sqrt{\hat{\hat{H}}}$ is T-ABSO F. ideal of R. 


\section{Lemma 4.}

Let $\hat{H} \subseteq \mathrm{P}$ be $\mathrm{F}$. ideal of a ring $\mathrm{R}$, where $\mathrm{P}$ is a prime $\mathrm{F}$. ideal. Then the following expressions are equivalent:

1- $\mathrm{P}$ is a minimal prime $\mathrm{F}$. ideal of $\hat{\mathrm{H}}$;

2- For each F. singleton $a_{v} \subseteq \mathrm{P}$, there exists F. singleton $b_{l}$ of $\mathrm{R} \backslash \mathrm{P}$ and a non-negative integer $n$ such that $b_{l} a_{v}^{n} \subseteq \hat{\mathrm{H}}, \forall v, l \in \mathrm{L}$.

Proof. (1) $\Rightarrow$ (2) Let $\mathrm{P}$ be a minimal prime F. ideal of $\hat{\mathrm{H}}$ and $a_{v} \subseteq \mathrm{P}$, suppose that for every $\mathrm{F}$. singleton $b_{l}$ of $\mathrm{R} \backslash \mathrm{P}, b_{l} a_{v}^{n} \nsubseteq \hat{\mathrm{H}}, \forall n \in N$. Inparticular, $a_{v}^{n} \nsubseteq \hat{\mathrm{H}}, \forall n \in N$.

Let $A=\left\{1, a_{v}, a_{v}^{2}, \ldots\right\}$ and $B=\{K: K$ is $\mathrm{F}$. ideal of $\mathrm{R}$ such that $K \cap A=\emptyset, \hat{\mathrm{H}} \subseteq K \subseteq \mathrm{P}\}$. Then $\mathrm{B} \neq \emptyset$, since $\hat{\mathrm{H}} \subseteq B$, it is obvious $\mathrm{B}$ is partially ordered by inclusion. By [13], $B$ has a maximal $\mathrm{F}$. ideal say $U_{x}$. Then $U_{\Lambda}$ is a prime F. ideal by [12], such that $\hat{H} \subseteq U \subseteq P$. Since $P$ is a minimal prime $F$. ideal of $\hat{\mathrm{H}}$, so $\mathrm{U}=\mathrm{P}$ this is a contradiction to $a_{v} \subseteq \mathrm{P}=\mathrm{U}$, hence $b_{l} a_{v}^{n} \subseteq \hat{\mathrm{H}}$.

(2) $\Rightarrow$ (1) Suppose that for each F. singleton $a_{v} \subseteq \mathrm{P}$, there exists F. singleton $b_{l}$ of $\mathrm{R} \backslash \mathrm{P}$ and $\mathrm{n} \in \mathrm{N}$ such that $b_{l} a_{v}^{n} \subseteq \hat{\mathrm{H}}$. Let $K$ be a prime F. ideal of $\mathrm{R}$ such that $\hat{\mathrm{H}} \subseteq K \subseteq \mathrm{P}$.

We claim that $\mathrm{P} \subseteq K$. Since $a_{v} \subseteq \mathrm{P}$, then there exists F. singleton $b_{l} \subseteq \mathrm{R} \backslash \mathrm{P}$ and $n \in N$ such that $b_{l} a_{v}^{n} \subseteq \hat{\mathrm{H}} \subseteq K$. Since $\mathrm{K}$ is a prime $\mathrm{F}$. ideal, then either $b_{l} \subseteq \mathrm{K}$ or $a_{v}^{n} \subseteq K$. Hence $a_{v} \subseteq K$ as $b_{l} \subseteq$ $\mathrm{R} \backslash \mathrm{P}$. So that $\mathrm{P} \subseteq K$, then $\mathrm{P}=K$; that is $\mathrm{P}$ is a minimal prime $\mathrm{F}$. ideal of $\hat{\mathrm{H}}$.

\section{Proposition 5.}

Suppose that $\hat{H}$ is T-ABSO F. ideal of a ring R. Then there are at most two prime F ideals of $\mathrm{R}$ that are minimal over $\hat{\mathrm{H}}$.

Proof. Assume that $K=\left\{\mathrm{P}_{i}: \mathrm{P}_{i}\right.$ is a prime $\mathrm{F}$. ideal of $\mathrm{R}$ which is minimal over $\left.\hat{\mathrm{H}}\right\}$. Let $K$ have at least three prime $\mathrm{F}$. ideals. Let $\mathrm{P}_{1}, \mathrm{P}_{2} \in K$ be two different prime $\mathrm{F}$. ideals. Then there exists $\mathrm{F}$. singleton $a_{s} \subseteq \mathrm{P}_{1} \backslash \mathrm{P}_{2}$ and there exists $\mathrm{F}$ singleton $b_{l} \subseteq \mathrm{P}_{2} \backslash \mathrm{P}_{1}$.

We show that $a_{s} b_{l} \subseteq \hat{\mathrm{H}}$. By lemma (4), there exist F. singletons $x_{v} \nsubseteq \mathrm{P}_{1}$ and $y_{h} \nsubseteq \mathrm{P}_{2}$, such that $x_{v} a_{s}^{n} \subseteq \hat{\mathrm{H}}$ and $y_{h} b_{l}^{m} \subseteq \hat{\mathrm{H}}$ for some $n, m \geq 1$. Since $\hat{\mathrm{H}}$ is T-ABSO F. ideal of R, we have $x_{v} a_{s} \subseteq \hat{\mathrm{H}}$ and $y_{h} b_{l} \subseteq \hat{\mathrm{H}}$. Since $a_{s}, b_{l} \nsubseteq \mathrm{P}_{1} \cap \mathrm{P}_{2}$ and $x_{v} a_{s}, y_{h} b_{l} \subseteq \hat{\mathrm{H}} \subseteq \mathrm{P}_{1} \cap \mathrm{P}_{2}$, we get $x_{v} \subseteq \mathrm{P}_{2} \backslash \mathrm{P}_{1}$ and $y_{h} \subseteq \mathrm{P}_{1} \backslash \mathrm{P}_{2}$, thus $x_{v}, y_{h} \nsubseteq \mathrm{P}_{1} \cap \mathrm{P}_{2}$. Since $x_{v} a_{s} \subseteq \hat{\mathrm{H}}$ and $y_{h} b_{l} \subseteq$ $\hat{\mathrm{H}}$, have $\left(x_{v}+y_{h}\right) a_{s} b_{l} \subseteq \hat{\mathrm{H}}$. Observe that $\left(x_{v}+y_{h}\right) \nsubseteq \mathrm{P}_{1}$ and $\left(x_{v}+y_{h}\right) \nsubseteq \mathrm{P}_{2}$. Since $\left(x_{v}+\right.$ $\left.y_{h}\right) a_{s} \nsubseteq \mathrm{P}_{2}$ and $\left(x_{v}+y_{h}\right) b_{l} \nsubseteq \mathrm{P}_{1}$, we conclude that neither $\left(x_{v}+y_{h}\right) a_{s} \subseteq \hat{\mathrm{H}}$ nor $\left(x_{v}+\right.$ $\left.y_{h}\right) b_{l} \subseteq \hat{\mathrm{H}}$ and hence $a_{s} b_{l} \subseteq \hat{\mathrm{H}}$. Now, suppose there exists $\mathrm{P}_{3} \in K$ such that $\mathrm{P}_{3}$ is neither $\mathrm{P}_{1}$ nor $\mathrm{P}_{2}$. Then we can choose $r_{k} \subseteq \mathrm{P}_{1} \backslash\left(\mathrm{P}_{2} \cup \mathrm{P}_{3}\right), c_{n} \subseteq \mathrm{P}_{2} \backslash\left(\mathrm{P}_{1} \cup \mathrm{P}_{3}\right)$ and $d_{m} \subseteq \mathrm{P}_{3} \backslash\left(\mathrm{P}_{1} \cup\right.$ $\mathrm{P}_{2}$ ). By the same way we show that $r_{k} c_{n} \subseteq \hat{\mathrm{H}}$. Since $\hat{\mathrm{H}} \subseteq \mathrm{P}_{1} \cap \mathrm{P}_{2} \cap \mathrm{P}_{3}$ and $r_{k} c_{n} \subseteq \hat{\mathrm{H}}$, we get either $r_{k} \subseteq \mathrm{P}_{3}$ or $c_{n} \subseteq \mathrm{P}_{3}$ this is a discrepancy. Hence $K$ has at most two prime F. ideals of $\mathrm{R}$.

\section{Proposition 6}

Let $\mathrm{H}$ be T-ABSO F. ideal of R. Then one of the following expressions must hold $1-\sqrt{\hat{H}}=P$ is a prime $F$. ideal of $R$ such that $P^{2} \subseteq \hat{H}$

2- $\sqrt{\hat{\mathrm{H}}}=\mathrm{P}_{1} \cap \mathrm{P}_{2}, \mathrm{P}_{1} \mathrm{P}_{2} \subseteq \hat{\mathrm{H}}$, and $(\sqrt{\hat{\mathrm{H}}})^{2} \subseteq \hat{\mathrm{H}}$ where $\mathrm{P}_{1}, \mathrm{P}_{2}$ are the only distinct prime $\mathrm{F}$. ideals of $\mathrm{R}$ that are minimal over $\hat{\mathrm{H}}$. 
Proof. By proposition (5), we get either $\sqrt{\hat{\mathrm{H}}}=\mathrm{P}$ is a prime $\mathrm{F}$. ideal of $\mathrm{R}$ or $\sqrt{\hat{\mathrm{H}}}=\mathrm{P}_{1} \cap \mathrm{P}_{2}$, where $P_{1}, P_{2}$ are the only distinct prime $F$. ideals of $R$ that are minimal over $\hat{H}$. Assume that $\sqrt{\hat{\mathrm{H}}}=\mathrm{P}$ is prime $\mathrm{F}$. ideal of $\mathrm{R}$. Let $\mathrm{F}$. singletons $a_{s}, b_{l} \subseteq \mathrm{P}$. By proposition (3), we have $a_{s}^{2}, b_{l}^{2} \subseteq \hat{\mathrm{H}}$. So that $a_{s}\left(a_{s}+b_{l}\right) b_{l} \subseteq \hat{\mathrm{H}}$. Since $\hat{\mathrm{H}}$ is T-ABSO F. ideal, we get $a_{s}\left(a_{s}+b_{l}\right)=$ $a_{s}^{2}+a_{s} b_{l} \subseteq \hat{\mathrm{H}}$ or $\left(a_{s}+b_{l}\right) b_{l}=a_{s} b_{l}+b_{l}^{2} \subseteq \hat{\mathrm{H}}$ or $a_{s} b_{l} \subseteq \hat{\mathrm{H}}$. From each case implies that $a_{v} b_{l} \subseteq \hat{\mathrm{H}}$, and so $\mathrm{P}^{2} \subseteq \hat{\mathrm{H}}$. Suppose that $\sqrt{\hat{\mathrm{H}}}=\mathrm{P}_{1} \cap \mathrm{P}_{2}$, where $\mathrm{P}_{1}, \mathrm{P}_{2}$ are the only distinct prime $\mathrm{F}$. ideals of $\mathrm{R}$ that are minimal over $\hat{\mathrm{H}}$. Let $\mathrm{F}$ singletons $a_{s}, b_{l} \subseteq \sqrt{\hat{\mathrm{H}}}$. By the same way of above, we have $a_{s} b_{l} \subseteq \hat{\mathrm{H}}$ and hence $(\sqrt{\hat{\mathrm{H}}})^{2} \subseteq \hat{\mathrm{H}}$. Now, we show that, $\mathrm{P}_{1} \mathrm{P}_{2} \subseteq \hat{\mathrm{H}}$. By proposition (3), we have $x_{v}^{2} \subseteq \hat{\mathrm{H}}$ for each $\mathrm{F}$ singleton $x_{v} \subseteq \sqrt{\hat{\mathrm{H}}}$. Let be $\mathrm{F}$ singleton $y_{h} \subseteq$ $\mathrm{P}_{1} \backslash \mathrm{P}_{2}$ and $r_{k} \subseteq \mathrm{P}_{2} \backslash \mathrm{P}_{1}$. By the proof of proposition (5), we have $y_{h} r_{k} \subseteq \hat{\mathrm{H}}$. Let $\mathrm{F}$ singletons $c_{n} \subseteq \sqrt{\hat{\mathrm{H}}}$ and $d_{m} \subseteq \mathrm{P}_{2} \backslash \mathrm{P}_{1}$, choose F singleton $f_{u} \subseteq \mathrm{P}_{1} \backslash \mathrm{P}_{2}$. Then $f_{u} d_{m} \subseteq \hat{\mathrm{H}}$ by the proof of proposition (5) and $\left(c_{n}+f_{u}\right) \subseteq \mathrm{P}_{1} \backslash \mathrm{P}_{2}$. Thus $c_{n} d_{m}+f_{u} d_{m}=\left(c_{n}+f_{u}\right) d_{m} \subseteq \hat{\mathrm{H}}$. So that $c_{n} d_{m} \subseteq \hat{\mathrm{H}}$. By the same method we show that if $c_{n} \subseteq \sqrt{\hat{\mathrm{H}}}$ and $d_{m} \subseteq \mathrm{P}_{1} \backslash \mathrm{P}_{2}$. Thus $\mathrm{P}_{1} \mathrm{P}_{2} \subseteq \hat{\mathrm{H}}$.

\section{Proposition 7}

Let $\hat{\mathrm{H}}$ be T-ABSO F. ideal of $\mathrm{R}$ such that $\sqrt{\hat{\mathrm{H}}}=\mathrm{P}$ is a prime F. ideal, of Rand suppose that $\hat{\mathrm{H}} \neq \mathrm{P}$. For each F. singleton $a_{v} \subseteq \mathrm{P} \backslash \hat{\mathrm{H}}$, let $A_{a_{v}}=\left\{b_{l} \subseteq R: b_{l} a_{v} \subseteq \hat{\mathrm{H}}\right\}, \forall v, l \in \mathrm{L}$. Then $A_{a_{v}}$ is a prime F. ideal of $\mathrm{R}$ included P. Futhermore, either $A_{b_{l}} \subseteq A_{a_{v}}$ or $A_{a_{v}} \subseteq A_{b_{l}}$ for each $\mathrm{F}$. singletons $a_{v}, b_{l} \subseteq \mathrm{P} \backslash \hat{\mathrm{H}}$.

Proof. Let $a_{v} \subseteq \mathrm{P} \backslash \hat{\mathrm{H}}$. Since $\mathrm{P}^{2} \subseteq \hat{\mathrm{H}}$ by proposition (6), we have $\mathrm{P} \subseteq A_{a_{v}}$. Assume that $\mathrm{P} \neq A_{a_{v}}$ and $b_{l} r_{k} \subseteq A_{a_{v}}$ for some F. singleton $b_{l}, r_{k}$ of R. Since $\mathrm{P} \subseteq A_{a_{v}}$, we may suppose that $b_{l} \nsubseteq \mathrm{P}$ and $r_{k} \nsubseteq \mathrm{P}$, hence $b_{l} r_{k} \nsubseteq \hat{\mathrm{H}}$. Since $b_{l} r_{k} \subseteq A_{a_{v}}$ we have $b_{l} r_{k} a_{v} \subseteq \hat{\mathrm{H}}$. Since $\hat{\mathrm{H}}$ is T-ABSO F. ideal of $\mathrm{R}$ and $b_{l} r_{k} \nsubseteq \hat{\mathrm{H}}$, we have either $b_{l} a_{v} \subseteq \hat{\mathrm{H}}$ or $r_{k} a_{v} \subseteq \hat{\mathrm{H}}$, thus either $b_{l} \subseteq A_{a_{v}}$ or $r_{k} \subseteq$ $A_{a_{v}}$. Hence $A_{a_{v}}$ is a prime F. ideal of $\mathrm{R}$ included P. Now, let $a_{v}, b_{l} \subseteq \mathrm{P} \backslash \hat{\mathrm{H}}$ for F. singletons $a_{v}, b_{l}$ of $\mathrm{R}$ and assume that $\mathrm{F}$ singleton $r_{k} \subseteq A_{a_{v}} \backslash A_{b_{l}}$. Since $\mathrm{P} \subseteq A_{b_{l}}$, so $r_{k} \subseteq A_{a_{v}} \backslash \mathrm{P}$. We show that $A_{b_{l}} \subseteq A_{a_{v}}$. Let $\mathrm{F}$ singleton $x_{s}$ of $\mathrm{R}$ such that $x_{s} \subseteq A_{b_{l}}$. Since $\mathrm{P} \subseteq A_{a_{v}}$, we may suppose that $x_{s} \subseteq A_{b_{l}} \mathrm{P}$. Since $r_{k} \nsubseteq \mathrm{P}$ and $x_{s} \nsubseteq \mathrm{P}$, we have $r_{k} x_{s} \nsubseteq \hat{\mathrm{H}}$. Since $r_{k}\left(a_{v}+b_{l}\right) x_{s} \subseteq$ $\hat{\mathrm{H}}$ and $r_{k} x_{s}, r_{k} b_{l} \nsubseteq \hat{\mathrm{H}}$, we have $\left(a_{v}+b_{l}\right) x_{s} \subseteq \hat{\mathrm{H}}$. Hence $a_{v} x_{s} \subseteq \hat{\mathrm{H}}$ since $\left(a_{v}+b_{l}\right) x_{s} \subseteq \hat{\mathrm{H}}$ and $x_{s} b_{l} \subseteq \hat{\mathrm{H}}$. Hence $x_{s} \subseteq A_{a_{v}}$. So that $A_{b_{l}} \subseteq A_{a_{v}}$.

Proposition 8. Assume that $\hat{H}$ is $F$. ideal of $R$ such that $\hat{H} \neq \sqrt{\hat{H}}$ and $\sqrt{\hat{H}}$ is a prime F. ideal of $\mathrm{R}$. Then the following expressions are equivalent:

$1-\hat{H}$ is T-ABSO F. ideal of $\mathrm{R}$;

2- $A_{a_{v}}=\left\{b_{l} \subseteq R: b_{l} a_{v} \subseteq \hat{\mathrm{H}}\right\}, \forall v, l \in \mathrm{L}$, is a prime F. ideal of $\mathrm{R}$ for each F. singleton $a_{v} \subseteq \sqrt{\hat{\mathrm{H}}} \backslash$ $\hat{\mathrm{H}}$. 
Proof. (1) $\Rightarrow(2)$ This is obvious by proposition (7).

(2) $\Rightarrow$ (1) Assume that $a_{v} b_{l} r_{k} \subseteq \hat{\mathrm{H}}$ for F. singletons $a_{v}, b_{l}, r_{k}$ of R.

Since $\sqrt{\hat{\mathrm{H}}}$ is a prime $\mathrm{F}$. ideal of $\mathrm{R}$, we may suppose that $a_{v} \subseteq \sqrt{\hat{\mathrm{H}}}$.

If $a_{v} \subseteq \hat{\mathrm{H}}$, then $a_{v} b_{l} \subseteq \hat{\mathrm{H}}$. Thus suppose that $\left.a_{v} \subseteq \sqrt{\hat{\mathrm{H}}}\right) \backslash \hat{\mathrm{H}}$. Hence $b_{l} r_{k} \subseteq A_{a_{v}}$. But $A_{a_{v}}$ is a prime F. ideal of $\mathrm{R}$, then by proposition (7), either $b_{l} a_{v} \subseteq \hat{\mathrm{H}}$ or $r_{k} a_{v} \subseteq \hat{\mathrm{H}}$. Thus $\hat{\mathrm{H}}$ is TABSO F. ideal of R.

\section{Proposition 9.}

Assume that $\hat{\mathrm{H}}$ is a non-constant proper $\mathrm{F}$. ideal of a ring $\mathrm{R}$. Then the following expressions are equivalent:

$1-\hat{\mathrm{H}}$ is T-ABSO F. ideal of R;

2- If $\mathrm{U} K T \subseteq \hat{\mathrm{H}}$ for F. ideals $\mathrm{U}, K, T$ of $\mathrm{R}, \quad \mathrm{U} K \subseteq \hat{\mathrm{H}}$ or $K T \subseteq \hat{\mathrm{H}}$ or $\mathrm{U} T \subseteq \hat{\mathrm{H}}$.

Proof. (1) $\Rightarrow(2)$ Assume that $\mathrm{U} K T \subseteq \hat{\mathrm{H}}$ for F. ideals $\mathrm{U}, K, T$ of R. By proposition (5), we have $\sqrt{\hat{\mathrm{H}}}$ is a prime $\mathrm{F}$. ideal of $\mathrm{R}$ or $\sqrt{\hat{\mathrm{H}}}=\mathrm{P}_{1} \cap \mathrm{P}_{2}$ where $\mathrm{P}_{1}, \mathrm{P}_{2}$ are non-constant distinct prime $\mathrm{F}$. ideals of $\mathrm{R}$ that are minimal over $\hat{\mathrm{H}}$. If $\hat{\mathrm{H}}=\sqrt{\hat{\mathrm{H}}}$, then it is readily showed that, $\mathrm{U} K \subseteq \hat{\mathrm{H}}$ or $K T \subseteq$ $\hat{\mathrm{H}}$ or $\mathrm{U} T \subseteq \hat{\mathrm{H}}$. Thus suppose that $\hat{\mathrm{H}} \neq \sqrt{\hat{\mathrm{H}}}$. We see the following:

(1) Assume that $\sqrt{\hat{\mathrm{H}}}$ is a prime F. ideal of R. Then we perhaps suppose that $U \subseteq \sqrt{\hat{\mathrm{H}}}$ andU $\nsubseteq$ $\hat{\mathrm{H}}$. Let F. singleton $a_{v}$ of R such that $a_{v} \subseteq \mathrm{U} \backslash \hat{\mathrm{H}}$. Since $a_{v} K T \subseteq \hat{\mathrm{H}}$, we have $K T \subseteq A_{a_{v}}$ where $A_{a_{v}}=\left\{b_{l} \subseteq R: b_{l} a_{v} \subseteq \hat{\mathrm{H}}\right\}$. Since $A_{a_{v}}$ is a prime F. ideal of $\mathrm{R}$ by proposition (8), we have either $K \subseteq A_{a_{v}}$ or $T \subseteq A_{a_{v}}$. If $K \subseteq A_{x_{s}}$ and $T \subseteq A_{x_{s}}$ for each F. singleton $x_{s} \subseteq \mathrm{U} \backslash \hat{\mathrm{H}}$, then $\mathrm{U} K \subseteq \hat{\mathrm{H}}$ (and $\mathrm{U} T \subseteq \hat{\mathrm{H}}$ ) and we are finished. Hence suppose that $K \subseteq A_{r_{k}}$ and $T \nsubseteq A_{r_{k}}$ for some F. singleton $r_{k} \subseteq \mathrm{U} \backslash \hat{\mathrm{H}}$. Since $\left\{A_{w_{h}}: w_{h} \subseteq \mathrm{U} \backslash \hat{\mathrm{H}}\right\}$, is a set of prime F. ideals of $\mathrm{R}$ that are linearly ordered by proposition (7), since $K \subseteq A_{r_{k}}$ and $T \nsubseteq A_{r_{k}}$, we have $K \subseteq A_{z_{n}}$ for some F. singleton $z_{n} \subseteq \mathrm{U} \backslash \hat{\mathrm{H}}$. ThusU $K \subseteq \hat{\mathrm{H}}$.

(2) Assume that $\sqrt{\hat{\mathrm{H}}}=\mathrm{P}_{1} \cap \mathrm{P}_{2}$ where $\mathrm{P}_{1}, \mathrm{P}_{2}$ are non-constant distinct prime $\mathrm{F}$. ideals of $\mathrm{R}$ that are minimal over $\hat{H}$. We suppose that $\mathrm{U} \subseteq \mathrm{P}$. If either $K \subseteq \mathrm{P}_{2}$ or $T \subseteq \mathrm{P}_{2}$, then either $\mathrm{U} K \subseteq \hat{\mathrm{H}}$ or $\mathrm{U} T \subseteq \hat{\mathrm{H}}$ because $\mathrm{P}_{1} \mathrm{P}_{2} \subseteq \hat{\mathrm{H}}$ by proposition (6). Hence suppose that $\mathrm{U} \subseteq \sqrt{\hat{\mathrm{H}}}$ andU $\nsubseteq \hat{\mathrm{H}}$. By the same way in (1) and by proposition (7), we are finished from this proof.

$(2) \Rightarrow(1)$ it is trivial. Now, we give the concept of T-ABSO quasi primary F. ideal as follows:

\section{Definition 10.}

A proper F. ideal $\hat{H}$ of $R$ is called T-ABSO quasi primary F. ideal of $R$ if $\sqrt{\hat{H}}$ is T-ABSO F. ideal of $\mathrm{R}$.

\section{Proposition 11.}

A proper $F$. ideal $\hat{H}$ of $R$ is T-ABSO quasi primary F. of $R$ iff whenever for each $F$. singleton $a_{s}, b_{l}, r_{h}$ of $\mathrm{R}, \forall s, l, h \in \mathrm{L}$, such that $a_{s} b_{l} r_{h} \subseteq \hat{\mathrm{H}}$, then $a_{s} b_{l} \subseteq \sqrt{\hat{\mathrm{H}}}$ or $a_{s} r_{h} \subseteq \sqrt{\hat{\mathrm{H}}}$ or $b_{l} r_{h} \subseteq \sqrt{\hat{\mathrm{H}}}$. 
Proof. $(\Leftarrow)$ Suppose that $\hat{H}$ is a proper F. ideal of $\mathrm{R}$ and whenever for each $\mathrm{F}$. singleton $a_{s}, b_{l}, r_{h}$ of $\mathrm{R}$, such that $a_{s} b_{l} r_{h} \subseteq \hat{\mathrm{H}}$, then $a_{s} b_{l} \subseteq \sqrt{\hat{\mathrm{H}}}$ or $a_{s} r_{h} \subseteq \sqrt{\hat{\mathrm{H}}}$ or $b_{l} r_{h} \subseteq \sqrt{\hat{\mathrm{H}}}$. Let $a_{s} b_{l} r_{h} \subseteq \sqrt{\hat{\mathrm{H}}}, a_{s} r_{h} \nsubseteq \sqrt{\hat{\mathrm{H}}}$ and $b_{l} r_{h} \nsubseteq \sqrt{\hat{\mathrm{H}}}$. Since $a_{s} b_{l} r_{h} \subseteq \sqrt{\hat{\mathrm{H}}}$, then there exists $n \in Z^{+}$such tha $\left(a_{s} b_{l} r_{h}\right)^{n}=a_{s}^{n} b_{l}^{n} r_{h}^{n} \subseteq \hat{\mathrm{H}}$. Since $a_{s}^{n} r_{h}^{n} \nsubseteq \hat{\mathrm{H}}$ and $b_{l}^{n} r_{h}^{n} \nsubseteq \hat{\mathrm{H}}$, then we have $a_{s}^{n} b_{l}^{n}=$ $\left(a_{s} b_{l}\right)^{n} \subseteq \hat{\mathrm{H}}$. So that $a_{s} b_{l} \subseteq \sqrt{\hat{\mathrm{H}}}$. Thus $\sqrt{\hat{\mathrm{H}}}$ is T-ABSO F. ideal of R and so that $\hat{\mathrm{H}}$ is TABSO quasi primary $F$. of R.

$\Leftrightarrow$ Let $\hat{\mathrm{H}}$ be T-ABSO quasi primary F. ideal of $\mathrm{R}$ and for each F. singleton $a_{s}, b_{l}, r_{h}$ of $\mathrm{R}$, such that $a_{s} b_{l} r_{h} \subseteq \hat{\mathrm{H}}$. Since $\hat{\mathrm{H}} \subseteq \sqrt{\hat{\mathrm{H}}}$ and $\sqrt{\hat{\mathrm{H}}}$ is T-ABSO F. ideal of R. So that $a_{s} b_{l} \subseteq \sqrt{\hat{\mathrm{H}}}$ or $a_{s} r_{h} \subseteq \sqrt{\hat{\mathrm{H}}}$ or $b_{l} r_{h} \subseteq \sqrt{\hat{\mathrm{H}}}$. The proposition specificities T-ABSO quasi primary F. ideal in terms of its level ideal is given as follow

\section{Proposition 12.}

A F. ideal $\hat{\mathrm{H}}$ of $\mathrm{R}$ is $\mathrm{T}$-ABSO quasi primary $\mathrm{F}$. iff the level ideal $\hat{\mathrm{H}}_{v}$ is $\mathrm{T}$-ABSO quasi primary ideal of $\mathrm{R}, \forall v \in \mathrm{L}$.

Proof. $(\Longrightarrow)$ Let $a b r \in \hat{\mathrm{H}}_{v}$ for each $a, b, r \in \mathrm{R}$ then $\hat{\mathrm{H}}(a b r) \geq v$ hence $(a b r)_{v} \subseteq \hat{\mathrm{H}}$. So that $a_{s} b_{l} r_{k} \subseteq \hat{\mathrm{H}}$ where $v=\min \{s, l, k\}$. Since $\hat{\mathrm{H}}$ is T-ABSO quasi primary F., then either $a_{s} b_{l} \subseteq \sqrt{\hat{\mathrm{H}}}$ or $a_{s} r_{k} \subseteq \sqrt{\hat{\mathrm{H}}}$ or $b_{l} r_{k} \subseteq \sqrt{\hat{\mathrm{H}}}$ hence either $(a b)_{v} \subseteq \sqrt{\hat{\mathrm{H}}}$ or $(a r)_{v} \subseteq \sqrt{\hat{\mathrm{H}}}$ or $(b r)_{v} \subseteq \sqrt{\hat{\mathrm{H}}}$ and so $a b \in \sqrt{\hat{\mathrm{H}}_{v}}$ or $a r \in \sqrt{\hat{\mathrm{H}}_{v}}$ or $b r \in \sqrt{\hat{\mathrm{H}}_{v}}$. Thus $\hat{\mathrm{H}}_{v}$ is T-ABSO quasi primary ideal of R.

$(\Leftarrow)$ Let $a_{s} b_{l} r_{k} \subseteq \hat{\mathrm{H}}$ for $\mathrm{F}$. singletons $a_{s}, b_{l}, r_{k}$ of R, $\forall s, l, k \in \mathrm{L}$. Hence $(a b r)_{v} \subseteq A$, where $v=\min \{s, l, k\}$, so that $\hat{\mathrm{H}}(a b r) \geq v$ and $a b r \in \hat{\mathrm{H}}_{v}$. But $\hat{\mathrm{H}}_{v}$ is T-ABSO quasi primary ideal then either $a b \in \sqrt{\hat{\mathrm{H}}_{v}}$ or $a r \in \sqrt{\hat{\mathrm{H}}_{v}}$ or $b r \in \sqrt{\hat{\mathrm{H}}_{v}}$, hence either $(a b)_{v} \subseteq \sqrt{\hat{\mathrm{H}}}$ or $(a r)_{v} \subseteq \sqrt{\hat{\mathrm{H}}}$ or $(b r)_{v} \subseteq \sqrt{\hat{\mathrm{H}}}$. So that either $a_{s} b_{l} \subseteq \sqrt{\hat{\mathrm{H}}}$ or $a_{s} r_{k} \subseteq \sqrt{\hat{\mathrm{H}}}$ or $b_{l} r_{k} \subseteq \sqrt{\hat{\mathrm{H}}}$. Thus $\hat{\mathrm{H}}$ is T-ABSO quasi primary $\mathrm{F}$. ideal of $\mathrm{R}$. The following theorem gives a characterization of T-ABSO quasi primary F. ideal.

\section{Theorem 13.}

Let $\hat{H}$ be a proper F. ideal of R. Then $\hat{H}$ is T-ABSO quasi primary F. ideal iff whenever $\mathrm{U} U T \subseteq \hat{\mathrm{H}}$ for some F. ideals $\mathrm{U}, K, T$ of $\mathrm{R}$, then $\mathrm{U} K \subseteq \sqrt{\hat{\mathrm{H}}}$ or $\mathrm{U} T \subseteq \sqrt{\hat{\mathrm{H}}}$ or $K T \subseteq \sqrt{\hat{\mathrm{H}}}$.Proof. $(\Leftarrow)$ Assume that $\mathrm{U} K T \subseteq \hat{\mathrm{H}}$ for some F. ideals $\mathrm{U}, K, T$ of $\mathrm{R}$, then $\mathrm{U} K \subseteq \sqrt{\hat{\mathrm{H}}}$ or $\mathrm{U} T \subseteq \sqrt{\hat{\mathrm{H}}}$ or $K T \subseteq \sqrt{\hat{\mathrm{H}}}$ and let $a_{s} b_{l} r_{k} \subseteq \hat{\mathrm{H}}$ for F. singleton $a_{s}, b_{l}, r_{k}$ of $\mathrm{R}$. Hence $<a_{s}><b_{l}><r_{k}>\subseteq$ $\hat{\mathrm{H}}$ and so that $\left\langle a_{s}><b_{l}>\subseteq \sqrt{\hat{\mathrm{H}}}\right.$ or $<a_{s}><r_{k}>\subseteq \sqrt{\hat{\mathrm{H}}}$ or $<b_{l}><r_{k}>\subseteq \sqrt{\hat{\mathrm{H}}}$. Then $a_{s} b_{l} \subseteq \sqrt{\hat{\mathrm{H}}}$ or $a_{s} r_{k} \subseteq \sqrt{\hat{\mathrm{H}}}$ or $b_{l} r_{k} \subseteq \sqrt{\hat{\mathrm{H}}}$. By proposition (11), then $\hat{\mathrm{H}}$ is T-ABSO quasi primary $F$. ideal of $R$.

$\Leftrightarrow$ ) Assume that $\hat{\mathrm{H}}$ is $\mathrm{T}$-ABSO quasi primary F. ideal of R and $\mathrm{U} K T \subseteq \hat{\mathrm{H}}$ for some F. ideals $\mathrm{U}, K, T$ of $\mathrm{R}$, then $\mathrm{U} K T \subseteq \sqrt{\hat{\mathrm{H}}}$. Since $\sqrt{\hat{\mathrm{H}}}$ is T-ABSO F. ideal of $\mathrm{R}$, then $\mathrm{U} K \subseteq \sqrt{\hat{\mathrm{H}}}$ or $\mathrm{U} T \subseteq$ $\sqrt{\hat{\mathrm{H}}}$ or $K T \subseteq \sqrt{\hat{\mathrm{H}}}$ by proposition (9). 


\section{T-ABSO F. Subm.}

In this section we present the concept of T-ABSO F. subm. and we introduce many basic properties and results about this concept.

\section{Definition 14.}

Let $X$ be F. M. of an R-M. $M$. A proper F. subm. $A$ of $X$ is called T-ABSO F. subm. if whenever $a_{s}, b_{l}$ be $\mathrm{F}$. singletons of $R$, and $x_{v} \subseteq X, \forall s, l, v \in L$ such that $a_{s} b_{l} x_{v} \subseteq A$, then either $a_{s} b_{l} \subseteq\left(A:_{R} X\right)$ or $a_{s} x_{v} \subseteq A$ or $b_{l} x_{v} \subseteq A$, see [7].

The proposition specificities T-ABSO F. subm. in terms of its level subm. is given as follow:

\section{Proposition 15.}

Let $A$ be T-ABSO F. subm. of F. M. $X$ of an R-M. $M$., iff the level subm. $A_{v}$ is T-ABSO subm. of $X_{v}$, for all $v \in \mathrm{L}$, see[7].

\section{Remarks and Examples}

1. The intersection of two distinct prime F. subms. of F. M. $X$ of an R-M, $M$ is T-ABSO F. subm.

Proof. Let $A$ and $B$ be two distinct prime F. subms. of $X$. Suppose that F. singletons $a_{s}, b_{l}$ of $\mathrm{R}$, $x_{v} \subseteq X$ such that $a_{s} b_{l} x_{v} \subseteq A \cap B$, but $a_{s} x_{v} \nsubseteq A \cap B$ and $b_{l} x_{v} \nsubseteq A \cap B$. Then $a_{s} x_{v} \nsubseteq A$, $b_{l} x_{v} \nsubseteq A, a_{s} x_{v} \nsubseteq B$ and $b_{l} x_{v} \nsubseteq B$ these are impossible since $A$ and $B$ are prime F. subms. So suppose that $a_{s} x_{v} \nsubseteq A$ and $b_{l} x_{v} \nsubseteq B$. Since $a_{s} b_{l} x_{v} \subseteq A$ and $a_{s} b_{l} x_{v} \subseteq B$, then $b_{l} \subseteq\left(A:_{R} X\right)$ and $a_{s} \subseteq\left(B:_{R} X\right)$. So that $a_{s} b_{l} \subseteq\left(A:_{R} X\right) \cap\left(B:_{R} X\right)=\left(A \cap B:_{R} X\right)$. Thus $A \cap B$ is T-ABSO F. subm. of $X$. (2). Every prime F. subm. is T-ABSO F. subm. Proof. Let $A$ be a prime F. subm. of F. M. $X$ of an R-M. $M$. Let $a_{s} b_{l} x_{k} \subseteq A$ for F. singletons $a_{s}, b_{l}$ of $\mathrm{R}$ and $x_{k} \subseteq X$. Then $(a b x)_{v} \subseteq A$ where $v=\min \{s, l, k\}$. Since $A$ is a proper subm. of $X$ then $A_{v}$ is a proper subm. of $X_{v}$, hence $A_{v}$ is prime subm. of $X_{v}$. So that $A_{v}$ is T-ABSO subm. (see [14]), hence $a b \in\left(A:_{R} X\right)_{v}$, then either $(a b)_{v} \subseteq\left(A:_{R} X\right)$ or $(a x)_{v} \subseteq A$ or $(b x)_{v} \subseteq A$. So either $a b \in$ $\left(A_{v}:_{R} X_{v}\right)$ or $a x \in A_{v}$ or $b x \in A_{v}$.

Since $\left(A_{v}:_{R} X_{v}\right)=\left(A:_{R} X\right)_{v}$ by [5]. So that Then either $a_{s} b_{l} \subseteq\left(A:_{R} X\right)$ or $a_{s} x_{k} \subseteq A$ or $b_{l} x_{k} \subseteq A$. Thus $A$ is T-ABSO F. subm. of $X$. However, the converse incorrect in general, for example:

Let $X: Z_{24} \rightarrow L$ such that $X(y)=\left\{\begin{array}{cc}1 & \text { if } y \in Z_{24} \\ 0 & \text { o.w. }\end{array}\right.$

It is obvious that $X$ is F. M. of $Z_{24}$ as $Z$-M.

Let $A: Z_{24} \rightarrow L$ such that $A(y)=\left\{\begin{array}{cc}v & \text { if } y \in(\overline{6}) \\ 0 & \text { o.w. }\end{array} \forall v \in L\right.$

It is obvious that $A$ is $\mathrm{F}$. subm. of $X$. Now $A_{v}=(\overline{6})$ is not prime subm. of $Z_{24}$, since $2 . \overline{3} \in$ $(\overline{6})$ but $\overline{3} \notin(\overline{6})$ and $2 \notin\left((\overline{6}):_{z} Z_{24}\right)=6 Z$. But $(\overline{6})=(\overline{2}) \cap(\overline{3})$ is T-ABSO subm.of $Z_{24}$ as $Z$-M. by [14]. So $A_{v}$ is T-ABSO subm., but not prime subm.,implies that $A$ is T-ABSO F. subm., but not prime F. subm. (3) It obvious every quasi-prime F. subm. is T-ABSO F. subm. However T-ABSO F. subm. may not be quasi-prime F.

subm. for example:

$$
\text { Let } X: Z \rightarrow \mathrm{L} \text { such that } X(y)=\left\{\begin{array}{cc}
1 & \text { if } y \in Z \\
0 & \text { o. } w
\end{array}\right.
$$


It is obvious that $X$ is F. M. of $Z$-M. $Z$.

Let $A: Z \rightarrow \mathrm{L}$ such that $A(y)=\left\{\begin{array}{cc}v & \text { if } y \in 6 Z \\ 0 & o . w .\end{array} \forall v \in L\right.$

It is obvious that $A$ is $\mathrm{F}$. subm. of $X$.

$A_{v}=6 \mathrm{Z}$ is $\mathrm{T}$-ABSO subm. of $Z$, since if $x, y, z \in Z$ and $x y z \in 6 Z=A_{v}$ then at least one of $x, y$ and $z$ is even or one of them is 6 . Then either $x y \in A_{v}$ or $x z \in A_{v}$ or $y z \in A_{v}$. But $6 Z=A_{v}$ is not quasi-prime, since $2.3 .1 \in 6 Z$, but $2.1 \notin 6 Z$ and $3.1 \notin 6 Z$. So

that $A$ is T-ABSO F. subm., but $A$ is not quasi-prime $\mathrm{F}$. subm. (4) Let $A, B$ be two F. subms. of F. M. $X$ of an R-M. $M$, and $B \subset A$. If $A$ is T-ABSO F. subm. of $X$, then it is not necessary that $B$ is a T-ABSO F. subm., for example:

Let $X: Z_{24} \rightarrow L$ such that $X(y)= \begin{cases}1 & \text { if } y \in Z_{24} \\ 0 & \text { o.w. }\end{cases}$

It is obvious that $X$ is F. M. of $Z-M . Z_{24}$.

Let $A: Z_{24} \rightarrow L$ such that $A(y)=\left\{\begin{array}{cc}v & \text { if } y \in(\overline{2}) \\ 0 & \text { o.w. }\end{array} \quad \forall v \in L\right.$

And $B: Z_{24} \rightarrow L$ such that $B(y)=\left\{\begin{array}{cc}v & \text { if } y \in(\overline{12}) \\ 0 & o . w .\end{array} \quad \forall v \in L\right.$

It is obvious that $A$ and $B$ are $\mathrm{F}$. subms. of $X$.

Now, $A_{v}=(\overline{2})$ and $B_{v}=(\overline{12})$ where $B_{v} \subset A_{v}$, since $A_{v}=(\overline{2})$ is maximal subm. of $Z_{24}$ as $Z$ M., then $A_{v}$ is prime subm. by[15]. Implies that $A_{v}$ is T-ABSO subm. by [14]. But 2.2. $\overline{3} \in B_{v}$, $2 . \overline{3} \notin B_{v}$ and $2.2=4 \notin\left(B_{v}: Z_{z} Z_{24}\right)=12 Z$. Thus $B_{v}$ is not T-A

BSO subm. Of $Z_{24}$ as Z-M. hence $B$ is not T-ABSO F. subm. (5) Let $A$ and $B$ be F. subms. of F. M. $X$ of an R-M . $M$ and $A \subset B$. If $A$ is T-ABSO F . subm. of $X$, then $A$ is T-ABSO F. subm. of $B$. Proof. If $B=X$, then don't need to prove. Let $a_{s} b_{l} x_{k} \subseteq A$ for F. singletons $a_{s}, b_{l}$ of $\mathrm{R}$ and $x_{k} \subseteq B$, implies $(a b x)_{v} \subseteq A$ hence $v=\min \{s, l, k\}$ $a b x \in A_{v}$, where $a, b \in \mathrm{R}, \quad x \in B_{v}$. Since $A \subset B$ implies where $A_{v} \subset B_{v}$. Since $A$ is T-ABS O F. subm. of $X$, then $A_{v}$ is T-ABSO subm. Of $X_{v}$. Hence $A_{v}$ is T-ABSO subm. Of $B_{v}$ by [14], so that either $a b \in\left(A_{v}:_{R} B_{v}\right) \rightarrow a b \in\left(A:_{R} B\right)_{v}$ or $a x \in A_{v}$ or $b x \in A_{v}$, then $(a b)_{v} \subseteq\left(A:_{R} B\right)$ or $(a x)_{v} \subseteq A$ or $(b x)_{v} \subseteq A$, implies either $a_{s} b_{l} \subseteq\left(A:_{R} B\right) \quad$ of T-A or $a_{s} x_{k} \subseteq A$ or $b_{l} x_{k} \subseteq A$. Thus $A$ is T-ABSO F. subm. of $B$. (6) The sum BSO F. subm. is not necessary TABSO F. subm., for example:

Let $X: Z \rightarrow$ L such that $X(y)=\left\{\begin{array}{cc}1 & \text { if } y \in Z \\ 0 & o . w .\end{array}\right.$

It is obvious that $X$ is F. M. of $Z$-M. $Z$.

Let $A: Z \rightarrow \mathrm{L}$ such that $A(y)=\left\{\begin{array}{lc}v & \text { if } y \in 2 Z \\ 0 & \text { o.w. }\end{array} \forall v \in L\right.$

It is obvious that $A$ is $\mathrm{F}$. subm. of $X$.

Let $B: \mathrm{Z} \rightarrow \mathrm{L}$ such that $B(y)=\left\{\begin{array}{lc}v & \text { if } y \in 3 Z \\ 0 & \text { o.w. }\end{array} \forall v \in L\right.$

It is obvious that $B$ is F. subm. of $X$. Now, $A_{v}=2 Z, B_{v}=3 Z$ where $A_{v}$ and $B_{v}$ be T- ABSO subms. of $Z$-M. $Z$, but $A_{v}+B_{v}=Z=X_{v}$ is not T-ABSO subm., implies that $A+B=X$ is not T-ABSO F. subm. (7) Let $A$ and $B$ be two F. subms. of F. M. $X$ of an R-M. $M$. If $A$ is T-ABSO F. subm. then it is not necessary that $B$ is T-ABSO F. subm., for example:

Let $X: Z \rightarrow$ L such that $X(y)= \begin{cases}1 & \text { if } y \in Z \\ 0 & o . w .\end{cases}$ 
It is obvious that $X$ is F. M. of $Z$-M. $Z$.

Let $A: Z \rightarrow \mathrm{L}$ such that $A(y)=\left\{\begin{array}{cc}v & \text { if } y \in 12 Z \\ 0 & o . w .\end{array} \forall v \in L\right.$

Let $B: \mathrm{Z} \rightarrow \mathrm{L}$ such that $B(y)=\left\{\begin{array}{cc}v & \text { if } y \in 10 Z \\ 0 & \text { o.w. }\end{array} \forall v \in L\right.$

It is obvious that $A$ and $B$ are F. subms. of $X$. Now, $A_{v}=2 Z, B_{v}=20 Z$ where $A_{v}$ is

T- ABSO subm. of $Z$ as Z-M., but $2 Z \cong 20 Z$ and $B_{v}=20 Z$ is not T-ABSO subm.

of $Z$ as $Z$-M. since $2.2 .5 \in B_{v}=20 Z$, but $2.5 \notin B_{v}=20 Z$ and $2.2 \notin B_{v}=20 Z$. Thus $A \cong B$ where $A$ is T- ABSO F. subm. of $X$ and $B$ is not T-ABSO F. subm. of $X$. (8) The intersection of two TABSO F. subms. need not be T-ABSO F. subm., for example:

Let $X: Z \rightarrow \mathrm{L}$ such that $X(y)= \begin{cases}1 & \text { if } y \in Z \\ 0 & \text { o. } w .\end{cases}$

It is obvious that $X$ is F. M. of $Z$-M. $Z$.

Let $A: Z \rightarrow \mathrm{L}$ such that $A(y)=\left\{\begin{array}{cc}v & \text { if } y \in 12 Z \\ 0 & o . w .\end{array} \forall v \in L\right.$

Let $B: Z \rightarrow \mathrm{L}$ such that $B(y)=\left\{\begin{array}{cc}v & \text { if } y \in 10 Z \\ 0 & o . w .\end{array} \forall v \in L\right.$

It is obvious that $A$ and $B$ are F. subms. of $X . A_{v}=12 Z, B_{v}=10 Z$ are T-ABSO subms. in the $Z$ as $Z$-M. But $A_{v} \cap B_{v}=12 Z \cap 10 Z=120 Z$ which is not T-ABSO since $2.6 .10 \in 120 Z$, but $2.10 \notin 120 Z$ and $6.10 \notin 120 Z$ and $2.6 \notin 120 Z$. Hence $A$ and $B$ subms., but $A \cap B$ is not T- ABSO F. subm (9) Let $A$ be T-ABSO are two T-ABSO F. subm. of F. M. $X$ of an R-M. $M$. Then for each $\mathrm{B} \subseteq X$, either $B \subseteq A$ or $B \cap A$ is T-ABSO F. subm. of $B$.

Proof. Assume that $B \nsubseteq A$ then $B \cap A \subsetneq B$ Let $a_{s}, b_{l}$ be F. singletons of $\mathrm{R}$ and $x_{k} \subseteq B$, such that $a_{s} b_{l} x_{k} \subseteq B \cap A$, implies $a_{s} b_{l} x_{k} \subseteq A$. Since $A$ is T-ABSO F. subm., thus either $a_{s} b_{l} \subseteq$ $\left(A:_{R} X\right)$ or $a_{s} x_{k} \subseteq A$ or $b_{l} x_{k} \subseteq A$. Then either $a_{s} b_{l} \subseteq\left(B \cap A:_{R} B\right)$ or $a_{s} x_{k} \subseteq B \cap A$ or $b_{l} x_{k} \subseteq B \cap A$. Thus $B \cap A$ is T-ABSO F. subm. of $B$.

\section{Proposition 17.}

Let $f: \dot{M}_{1} \rightarrow \bar{M}_{2}$ be an epimorphism, where $X_{1}, X_{2}$ are F. M. of R- M. $\dot{M}_{1}$ and $\dot{M}_{2}$ resp. If $B$ is T-ABSO F. subm. of $X_{2}$, then $f^{-1}(B)$ is T-ABSO F. subm. of $X_{1}$. Proof. Since $B$ is F. subm. of $X_{2}$, then $f^{-1}(B)$ is F. subm. of $X_{1}$, since $f$ is epimorphism. Let $a_{s} b_{l} x_{k} \subseteq f^{-1}(B)$ for F. singletons $a_{s}, b_{l}$ of $\mathrm{R}$ and $x_{k} \subseteq X_{1}$. Then $a_{s} b_{l} f\left(x_{k}\right) \subseteq B$ and since $B$ is TABSO F. subm., then either $a_{s} f\left(x_{k}\right) \subseteq B$ or $b_{l} f\left(x_{k}\right) \subseteq B$ or $a_{s} b_{l} \subseteq\left(B:_{R} X_{2}\right)$. Hence either $a_{s} x_{k} \subseteq f^{-1}(B)$ or $b_{l} f\left(x_{k}\right) \subseteq f^{-1}(B)$ or $a_{s} b_{l} X_{2} \subseteq B$. But $f\left(X_{1}\right) \subseteq X_{2}$, so that $a_{s} b_{l} f\left(X_{1}\right) \subseteq B$, hence $a_{s} b_{l} X_{1} \subseteq$ $f^{-1}(B)$, implies $a_{s} b_{l} \subseteq\left(f^{-1}(B):_{R} X_{1}\right.$ Thus $f^{-1}(B)$ is T-ABSO F. subm. of $X_{1}$

\section{Proposition 18.}

Let $f: \dot{\mathrm{M}}_{1} \rightarrow \mathrm{M}_{2}$ be an epimorphism, and $X_{1}, X_{2}$ are F. M. of R-M. $\dot{\mathrm{M}}_{1}$ and $\mathrm{M}_{2}$ resp. Let $A \subseteq X_{1}$ such that $F$-ker $f \subseteq A$. Then A is T-ABSO F. subm. of $X_{1}$ iff $f(A)$ is T-ABSO F. subm. of $X_{2}$.

Proof. $\Rightarrow$ Let $a_{s}, b_{l}$ be F. singletons of R and $y_{h} \subseteq X_{2}$ where $y_{h}=f\left(x_{k}\right)$ for some F. singleton $x_{k} \subseteq X_{1}$, such that $a_{s} b_{l} y_{h} \subseteq f(A)$. Hence $a_{s} b_{l} f\left(x_{k}\right) \subseteq f(A) a_{s} b_{l} f\left(x_{k}\right) \subseteq f(A)$ since $f$ is onto. Then $a_{s} b_{l} f\left(x_{k}\right)=f\left(z_{n}\right)$ for some F. singleton $z_{n} \subseteq A$. So that $f\left(a_{s} b_{l} x_{k}\right)=$ 
$f\left(z_{n}\right)$, hence $f\left(a_{s} b_{l} x_{k}\right)-f\left(z_{n}\right)=0_{1}$; that is $f\left(a_{s} b_{l} x_{k}-z_{n}\right)=0_{1}$, implies $a_{s} b_{l} x_{k}-z_{n} \subseteq$ $F-\operatorname{kerf} \subseteq A$.

So that $a_{s} b_{l} x_{k} \subseteq A$. Since $A$ is T-ABSO F. subm., then either $a_{s} b_{l} \subseteq\left(A:_{R} X_{1}\right)$ or $a_{s} x_{k} \subseteq A$ or $b_{l} x_{k} \subseteq A$. Hence either $a_{s} b_{l} X_{1} \subseteq A \rightarrow f\left(a_{s} b_{l} X_{1}\right) \subseteq f(A)$ or $f\left(a_{s} x_{k}\right) \subseteq f(A)$ or $f\left(b_{l} x_{k}\right) \subseteq f(A)$, implies either $a_{s} b_{l} f\left(X_{1}\right) \subseteq f(A) \rightarrow a_{s} b_{l} X_{2} \subseteq f(A)$ or $a_{s} f\left(x_{k}\right) \subseteq f(A)$ or $b_{l} f\left(x_{k}\right) \subseteq f(A)$. Then either $a_{s} b_{l} \subseteq\left(f(A):_{R} X_{2}\right)$ or $a_{s} y_{h} \subseteq f(A)$ or $b_{l} y_{h} \subseteq f(A)$. Thus $f$ $(A)$ is T-ABSO F. subm. of $X_{2}$.

$(\Leftrightarrow)$ Let $a_{s} b_{l} x_{k} \subseteq A$ for $\mathrm{F}$. singletons $a_{s}, b_{l}$ of $\mathrm{R}$ and $x_{k} \subseteq X_{1}$. Hence $f\left(a_{s} b_{l} x_{k}\right) \subseteq f(A)$, implies $a_{s} b_{l} f\left(x_{k}\right) \subseteq f(A)$. But $f(A)$ is T-ABSO F. subm., then either $a_{s} b_{l} \subseteq\left(f(A):_{R} X_{2}\right)$ or $a_{s} f\left(x_{k}\right) \subseteq f(A)$ or $b_{l} f\left(x_{k}\right) \subseteq f(A)$.

If $a_{s} b_{l} \subseteq\left(f(A): X_{2}\right)$, then $a_{s} b_{l} X_{2} \subseteq f(A)$, implies $a_{s} b_{l} f\left(X_{1}\right) \subseteq f(A)$ since $f$ is onto. Hence $f\left(a_{s} b_{l} X_{1}\right) \subseteq f(A)$, so that $a_{s} b_{l} X_{1} \subseteq A$; that is $a_{s} b_{l} \subseteq\left(A:_{R} X_{1}\right)$. If $a_{s} f\left(x_{k}\right) \subseteq$ $f(A)$ then $f\left(a_{s} x_{k}\right)=f\left(z_{n}\right)$ for some F. singleton $z_{n} \subseteq A, \forall n \in$ L. Hence $f\left(a_{s} x_{k}\right)-$ $f\left(z_{n}\right)=0_{1}$, implies $a_{s} x_{k}-z_{n} \subseteq F-k e r f \subseteq A$. So that $a_{s} x_{k} \subseteq A$. If $b_{l} f\left(x_{k}\right) \subseteq f(A)$ , then by the same way above, we have $b_{l} x_{k} \subseteq A$. Therefore, $A$ is T-ABSO F. subm. of $X_{1}$.

Proposition 19. Let $A$ be a proper F. subm. of F. M. $X$ of an R-M $M$. Then $A$ is T-ABSO F. subm. of $X$ iff $a_{s} b_{l} B \subseteq A$ for F. singletons $a_{s}, b_{l}$ of $\mathrm{R}$ and $B$ is $\mathrm{F}$. subm. of $X$ implies $a_{s} b_{l} \subseteq\left(A:_{R} X\right)$ or $a_{s} B \subseteq A$ or $b_{l} B \subseteq A$.

Proof. $(\Rightarrow)$ Let $A$ be T-ABSO F. subm. and $a_{s} b_{l} B \subseteq A$. Assume that $a_{s} b_{l} \nsubseteq(A: X), a_{s} B \nsubseteq A$ and $b_{l} B \nsubseteq A$. Then there exist F. singletons $x_{v}, y_{k} \subseteq B$, such that $a_{s} x_{v} \nsubseteq A$ and $b_{l} y_{k} \nsubseteq A$. Since $a_{s} b_{l} x_{v} \subseteq A$ and $a_{s} b_{l} \nsubseteq\left(A:_{R} X\right), a_{s} x_{v} \nsubseteq A$, we have $b_{l} x_{v} \subseteq A$. Also since $a_{s} b_{l} y_{k} \subseteq A$ and $a_{s} b_{l} \nsubseteq\left(A:_{R} X\right), b_{l} y_{k} \nsubseteq A$, we have $a_{s} y_{k} \subseteq A$. Now, since $a_{s} b_{l}\left(x_{v}+y_{k}\right) \subseteq A$ and $a_{s} b_{l} \nsubseteq$ $\left(A:_{R} X\right)$, we have $a_{s}\left(x_{v}+y_{k}\right) \subseteq A$ or $b_{l}\left(x_{v}+y_{k}\right) \subseteq A$. If $a_{s}\left(x_{v}+y_{k}\right) \subseteq A$, then $\left(a_{s} x_{v}+\right.$ $\left.a_{s} y_{k}\right) \subseteq A$ and since $a_{s} y_{k} \subseteq A$, we get $a_{s} x_{v} \subseteq A$, this is a discrepancy. If $b_{l}\left(x_{v}+y_{k}\right) \subseteq A$, then $\left(b_{l} x_{v}+b_{l} y_{k}\right) \subseteq A$ and since $b_{l} x_{v} \subseteq A$, we get $b_{l} y_{k} \subseteq A$ this is a discrepancy. Thus either $a_{s} b_{l} \subseteq\left(A:_{R} X\right)$ or $a_{s} B \subseteq A$ or $b_{l} B \subseteq A$.

$(\Leftarrow)$ It is obvious. The next theorem gives a characterization of T-ABSO F. subm.

\section{Theorem 20.}

Let $A$ be a proper F. subm. of F. M. $X$ of an R-M. $M$. Then the following expressions are equivalent:

$1-A$ is T-ABSO F. subm. of $X$;

2- If $\hat{\mathrm{H} U} U B \subseteq A$, for some $\mathrm{F}$. ideals $\hat{\mathrm{H}}, \mathrm{U}$ of $\mathrm{R}$ and $\mathrm{F}$. subm. $B$ of $X$, then either $\hat{\mathrm{H}} B \subseteq A$ or $\mathrm{U} B \subseteq A$ or $\hat{\mathrm{H}} \bigcup \underline{\Lambda} \subseteq\left(A:_{R} X\right)$.

Proof. (1) $\Rightarrow(2)$ Suppose that $A$ is T-ABSO F. subm. of $X$ and $\hat{\mathrm{H} U} U \subseteq \subseteq A$ for some F. ideals $\hat{\mathrm{H}}, \mathrm{U}$ of $\mathrm{R}$ and some F. subm. $B$ of $X$. Let $\hat{\mathrm{H} U} \nsubseteq\left(A:_{R} X\right)$, to prove $\hat{\mathrm{H}} B \subseteq A$ or $\mathrm{U} B \subseteq A$. Assume that $\hat{\mathrm{H}} B \nsubseteq A$ and $\mathrm{U} B \nsubseteq A$, then there exist $\mathrm{F}$. singletons $a_{s} \subseteq \hat{\mathrm{H}}$ and $b_{l} \subseteq \mathrm{U}$, such that $a_{s} B \nsubseteq A$ and $b_{l} B \nsubseteq A$. But $a_{s} b_{l} B \subseteq A$ and neither $a_{s} B \nsubseteq A$ nor $b_{l} B \nsubseteq A$ and A is T-ABSO F. subm., so that $a_{s} b_{l} \subseteq\left(A:_{R} X\right)$. Since $\hat{\mathrm{H} U} \Psi \nsubseteq\left(A:_{R} X\right)$, then there exist F. singletons $x_{v} \subseteq \hat{\mathrm{H}}$ and $y_{k} \subseteq \mathrm{U}$, such that $x_{v} y_{k} \nsubseteq\left(A:_{R} X\right)$. But $x_{v} y_{k} B \subseteq A$, so that $x_{v} B \subseteq A$ or $y_{k} B \subseteq A$ by proposition (19). 
Now we have the following:

(1) If $x_{v} B \subseteq A$ and $y_{k} B \nsubseteq A$, since $a_{s} y_{k} B \subseteq A$ and $y_{k} B \nsubseteq A, a_{s} B \nsubseteq A$, so that $a_{s} y_{k} \subseteq$ $\left(A:_{R} X\right)$ by proposition (19). Since $x_{v} B \subseteq A$ and $a_{s} B \nsubseteq A$, hence $\left(a_{s}+x_{v}\right) B \nsubseteq A$. On the other hand, $\left(a_{s}+x_{v}\right) y_{k} B \subseteq A$ and neither $\left(a_{s}+x_{v}\right) B \subseteq A$ nor $y_{k} B \subseteq A$, we get $\left(a_{s}+\right.$ $\left.x_{v}\right) y_{k} \subseteq\left(A:_{R} X\right)$ by proposition (19). But $\left(a_{s}+x_{v}\right) y_{k}=\left(a_{s} y_{k}+x_{v} y_{k}\right) \subseteq\left(A:_{R} X\right)$ and $a_{s} y_{k} \subseteq\left(A:_{R} X\right)$, we get $x_{v} y_{k} \subseteq\left(A:_{R} X\right)$ this is a discrepancy.

(2) If $y_{k} B \subseteq A$ and $x_{v} B \nsubseteq A$. By the same way of (1), we get a discrepancy.

(3) If $x_{v} B \subseteq A$ and $y_{k} B \subseteq A$. Since $y_{k} B \subseteq A$ and $b_{l} B \nsubseteq A$, we have $\left(b_{l}+y_{k}\right) B \nsubseteq A$. But $a_{s}\left(b_{l}+y_{k}\right) B \subseteq A$ and neither $a_{s} B \subseteq A$ nor $\left(b_{l}+y_{k}\right) B \subseteq A$. Thus $a_{s}\left(b_{l}+y_{k}\right) \subseteq$ $\left(A:_{R} X\right)$ by proposition (19). Since $a_{s} b_{l} \subseteq\left(A:_{R} X\right)$ and $\left(a_{s} b_{l}+a_{s} y_{k}\right) \subseteq\left(A:_{R} X\right)$, we get $a_{s} y_{k} \subseteq\left(A:_{R} X\right)$. Since $\left(a_{s}+x_{v}\right) b_{l} B \subseteq A$ and neither $b_{l} B \subseteq A$ nor $\left(a_{s}+x_{v}\right) B \subseteq A$, we have $\left(a_{s}+x_{v}\right) b_{l} \subseteq\left(A:_{R} X\right)$ by proposition (19). But $\left(a_{s}+x_{v}\right) b_{l}=\left(a_{s} b_{l}+x_{v} b_{l}\right) \subseteq\left(A:_{R} X\right)$ and since $a_{s} b_{l} \subseteq\left(A:_{R} X\right)$, we have $x_{v} b_{l} \subseteq\left(A:_{R} X\right)$. Now, since $\left(a_{s}+x_{v}\right)\left(b_{l}+y_{k}\right) B \subseteq A$ and neither $\left(a_{s}+x_{v}\right) B \subseteq A$ nor $\left(b_{l}+y_{k}\right) B \subseteq A$, we get $\left(a_{s}+x_{v}\right)\left(b_{l}+y_{k}\right) \subseteq\left(A:_{R} X\right)$ by proposition (19), where $\left(a_{s}+x_{v}\right)\left(b_{l}+y_{k}\right)=\left(a_{s} b_{l}+a_{s} y_{k}+x_{v} b_{l}+x_{v} y_{k}\right) \subseteq\left(A:_{R} X\right)$. But $\left(a_{s} b_{l}+a_{s} y_{k}+x_{v} b_{l}\right) \subseteq\left(A:_{R} X\right)$, so that $x_{v} y_{k} \subseteq\left(A:_{R} X\right)$ this is a discrepancy. Thus $\hat{\mathrm{HB} \subseteq \mathrm{A} \text { or }}$ $\mathrm{U} B \subseteq A$

(2) $\Rightarrow$ (1) It is obvious.

\section{Theorem 21.}

If $A$ is T-ABSO F. subm. of F. M. $X$ of an R-M. $M$, then $\left(A:_{R} X\right)$ is T-ABSO F. ideal of R. Proof. Let $a_{s} b_{l} r_{k} \subseteq\left(A_{R} X\right)$ for F. singletons $a_{s}, b_{l}, r_{k}$ of $\mathrm{R}$.

If $a_{s} r_{k} \nsubseteq\left(A:_{R} X\right)$ and $b_{l} r_{k} \nsubseteq\left(A:_{R} X\right)$, then there exist F. singletons $x_{v}, y_{h} \subseteq X \backslash A$, such that $a_{s} r_{k} x_{v} \nsubseteq A$ and $b_{l} r_{k} y_{h} \nsubseteq A$. Since $a_{s} b_{l}\left(r_{k}\left(x_{v}+y_{h}\right)\right) \subseteq A$ and A is T-ABSO F. subm., then either $a_{s} b_{l} \subseteq\left(A:_{R} X\right)$ or $a_{s} r_{k}\left(x_{v}+y_{h}\right) \subseteq A$ or $b_{l} r_{k}\left(x_{v}+y_{h}\right) \subseteq A$. If $a_{s} r_{k}\left(x_{v}+y_{h}\right) \subseteq A$ and since $a_{s} r_{k} x_{v} \nsubseteq A$, then we have $a_{s} r_{k} y_{h} \nsubseteq A$. So that $a_{s} b_{l}\left(r_{k} y_{h}\right) \subseteq A$ and $b_{l} r_{k} y_{h} \nsubseteq A$, hence $a_{s} b_{l} \subseteq\left(A:_{R} X\right)$. By the same method if $b_{l} r_{k}\left(x_{v}+y_{h}\right) \subseteq A$, we get $a_{s} b_{l} \subseteq\left(A:_{R} X\right)$.

Thus $\left(A:_{R} X\right)$ is T-ABSO F. ideal of $\mathrm{R}$.

\section{Theorem 22.}

Let $X$ be multiplication F. M. of an R-M. $M$, and $A$ is a proper F. subm. of $X$. If $\left(A:_{R} X\right)$ is T-ABSO F. ideal of R, then $A$ is T-ABSO F. subm. of $X$.

Proof. Let $a_{s} b_{l} x_{v} \subseteq A$ for F. singletons $a_{s}, b_{l}$ of $\mathrm{R}$ and $x_{v} \subseteq X$, then $a_{s} b_{l}<x_{v}>\subseteq A$. But $<$ $x_{v}>=\hat{\mathrm{H}} X$ for some F. ideal $\hat{\mathrm{H}}$ of $\mathrm{R}$ since $X$ is multiplication F. M., so that $a_{s} b_{l} \hat{\mathrm{H}} X \subseteq A$. Thus $a_{s} b_{l} \hat{\mathrm{H}} \subseteq\left(A:_{R} X\right)$, so we have that $<a_{s}><b_{l}>\hat{\mathrm{H}} \subseteq\left(A:_{R} X\right)$. Since $\left(A:_{R} X\right)$ is $\mathrm{T}$ ABSO F. ideal of $\mathrm{R}$, we get either $<a_{s}>\hat{\mathrm{H}} \subseteq\left(A:_{R} X\right)$ or $<b_{l}>\hat{\mathrm{H}} \subseteq\left(A:_{R} X\right)$ or $<a_{s}><$ $b_{l}>\subseteq\left(A:_{R} X\right)$ by

Proposition (9).

1) If $\left\langle a_{s}>\hat{\mathrm{H}} \subseteq\left(A:_{R} X\right)\right.$, then $<a_{s}>\hat{\mathrm{H}} X \subseteq A$ and so $<a_{s}><x_{v}>\subseteq A$. Hence $a_{s} x_{v} \subseteq$ A

2) If $\left\langle b_{l}\right\rangle \hat{\mathrm{H}} \subseteq\left(A:_{R} X\right)$, then by the same method $b_{l} x_{v} \subseteq A$.

3) If $\left.\left\langle a_{s}\right\rangle<b_{l}\right\rangle \subseteq\left(A:_{R} X\right)$, then $a_{s} b_{l} \subseteq\left(A:_{R} X\right)$.

By combining theorem (21) and theorem (22), we have the following corollary: 


\section{Corollary 23.}

Let $A$ be a proper F. subm. of a multiplication F. M. $X$ of an R-M. $M$. Then $A$ is T-ABSO F. subm. of $X$ iff $\left(A:_{R} X\right)$ is T-ABSO F. ideal of $\mathrm{R}$.

\section{Remark 24.}

The condition $X$ is multiplication F. M. can't be deleted from theorem (22). See the following example:

Let $X: Z_{p^{\infty}} \rightarrow L$ such that $X(y)=\left\{\begin{array}{cc}1 & \text { if } y \in Z_{p^{\infty}} \\ 0 & o . w .\end{array}\right.$

It is obvious that $X$ is F. M. of $Z$-M. $Z_{p^{\infty}}$.

Let $A: Z_{p^{\infty}} \rightarrow L$ such that $A(y)=\left\{\begin{array}{cc}v & \text { if } y \in(0) \\ 0 & \text { o.w. }\end{array} \quad \forall v \in L\right.$

It is obvious that $A$ is $\mathrm{F}$. subm. of $X$.

Now, $A_{v}=(0)$ is not T-ABSO subm. of $X_{v}=Z_{p^{\infty}}$, since $p^{2}<\frac{1}{p^{2}}+Z>=(0)$ but $p<\frac{1}{p^{2}}+$ $Z>\neq(0)$ and $p^{2} \notin\left((0):_{Z} Z_{p^{\infty}}\right)=0$. Note (0) is a prime ideal in $Z$, so that $\left((0):_{Z} Z_{p^{\infty}}\right)=0$ is T-ABSO ideal in $Z$; that is $\left(A_{v}:_{R} X_{v}\right)$ is T-ABSO ideal in $Z$, then $\left(A:_{R} X\right)$ is T-ABSO F. ideal in $Z$. Thus $A$ is not T-ABSO F. subm. of $X$, but $\left(A:_{R} X\right)$ is T-ABSO F. ideal in $Z$. Now, we gave the following theorem is a characterization of T-ABSO F. subm.

\section{Theoerm 25.}

Let $A$ be a proper F. subm. of a multiplication F. M. $X$ of $\dot{M}$. Then $A$ is T-ABSO F. subm. of $X$ iff $A_{1} A_{2} A_{3} \subseteq A$ implies that $A_{1} A_{2} \subseteq A$ or $A_{1} A_{3} \subseteq A$ or $A_{2} A_{3} \subseteq A$, where $A_{1}, A_{2}, A_{3}$ are F. subm. of $X$.

Proof. $\left(\Rightarrow\right.$ )Since $X$ is a multiplication F., then $A_{1}=\hat{\mathrm{H}} X, A_{2}=\mathrm{U} X$ and $A_{3}=K X$ for some $\mathrm{F}$. ideals $\hat{\mathrm{H}}, \mathrm{U}$ and $K$ of $\mathrm{R}$. So that the product of $A_{1}, A_{2}$ and $A_{3}$ as follows: $A_{1} A_{2} A_{3}=\hat{\mathrm{H} U} \mathrm{X} K X \subseteq A$. by [16]. Hence $\mathrm{H} U \mathrm{~N} K \subseteq\left(A:_{R} X\right)$. Since $A$ is T-ABSO F. subm. of X, then $\left(A:_{R} X\right)$ is T-ABSO F. ideal by theorem (21). So by proposition (9), either $\hat{\mathrm{H} U} \subseteq\left(A:_{R} X\right)$ or $\hat{\mathrm{H}} K \subseteq\left(A:_{R} X\right)$ or $\mathrm{U}_{\Lambda} K \subseteq\left(A:_{R} X\right)$. Hence either $\hat{\mathrm{H}} \mathrm{U}_{\Lambda} X \subseteq A$ or $\hat{\mathrm{H}} K X \subseteq A$ or $\mathrm{U}_{\Lambda} K X \subseteq A$, then $A_{1} A_{2} \subseteq A$ or $A_{1} A_{3} \subseteq A$ or $A_{2} A_{3} \subseteq A$.

$(\Leftarrow)$ Let $\hat{\mathrm{H}} \mathrm{U} B \subseteq A$ for some F. ideals $\mathrm{H}, \mathrm{U}$ of $\mathrm{R}$ and $B$ is F. subm. of $X$.

Since $X$ is a multiplication F. M., then $B=E X$ for some F. ideal $E$ of R. Then $\hat{\mathrm{H} U} E X \subseteq A$. Let

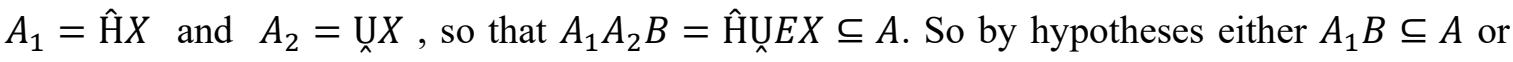
$A_{2} B \subseteq A$ or $A_{1} A_{2} \subseteq A$, hence $\hat{\mathrm{H}} E X \subseteq A$ or $\quad \mathrm{U} E X \subseteq A$ or $\hat{\mathrm{H} U} U X \subseteq A$. Thus $\hat{\mathrm{H}} B \subseteq A$ or $\mathrm{U} B \subseteq A$ or $\hat{\mathrm{H}} \mathrm{U} \subseteq\left(A:_{R} X\right)$. Therefore, $A$ is T-ABSO F. subm. of $X$ by theorem (20). Now, the definitions of finitely generated F. M. see [17, Definition (2.11)] and faithful F. M. see [3, Definition (3.2.6)]. We give the following proposition.

\section{Proposition 26.}

Let $X$ be a finitely generated multiplication F. M. of an R-M. $M$. If $\hat{\mathrm{H}}$ is T-ABSO F. ideal of $\mathrm{R}$ such that $F$-ann $X \subseteq \hat{\mathrm{H}}$, then $\hat{\mathrm{H}} X$ is T-ABSO F. subm. of $X$.

Proof. Let $a_{s} b_{l} x_{v} \subseteq \hat{\mathrm{H}} X$, where $a_{s}, b_{l}$ be F. singletons of $\mathrm{R}$ and $x_{v} \subseteq X$, hence $a_{s} b_{l}<x_{v}>$ $\subseteq \hat{\mathrm{H}} X$. But $X$ is a multiplication F. M., then $\left\langle x_{v}\right\rangle=\mathrm{U}_{X} X$ for some F. ideal $\mathrm{U}$ of R. Thus 
$a_{s} b_{l} \mathrm{U} X \subseteq \hat{\mathrm{H}} X$. So that $a_{s} b_{l} \mathrm{U} \subseteq \hat{\mathrm{H}}+F-a n n X=\hat{\mathrm{H}}$ since $F$-ann $X \subseteq \hat{\mathrm{H}}$. But $\hat{\mathrm{H}}$ is T-ABSO F. ideal of $\mathrm{R}$, so that either $a_{s} b_{l} \subseteq \hat{\mathrm{H}}$ or $a_{s} \mathrm{U} \subseteq \hat{\mathrm{H}} \quad b_{l} \mathrm{U} \subseteq \hat{\mathrm{H}}$. Then we have $a_{s} b_{l} X \subseteq \hat{\mathrm{H}} X$ or $a_{s} \mathrm{U} X \subseteq \hat{\mathrm{H}} X$ or $b_{l} \mathrm{U} X \subseteq \hat{\mathrm{H}} X$, so that $a_{s} b_{l} \subseteq\left(\hat{\mathrm{H}} X:_{R} X\right)$ or $a_{s}<x_{v}>\subseteq \hat{\mathrm{H}} X$ or $b_{l}<x_{v}>\subseteq$ $\hat{\mathrm{H}} X$, hence $a_{s} b_{l} \subseteq\left(\hat{\mathrm{H}} X:_{R} X\right)$ or $a_{s} x_{v} \subseteq \hat{\mathrm{H}} X$ or $b_{l} x_{v} \subseteq \hat{\mathrm{H}} X$. So that $\hat{\mathrm{H}} X$ is T-ABSO F. subm. of $X$.

\section{Corollary 27.}

Let $X$ be a faithful finitely generated multiplication F. M. of $M$. If $\hat{\mathrm{H}}$ is T-ABSO F. ideal of $\mathrm{R}$, then $\hat{\mathrm{H}} X$ is T-ABSO F. subm. of $X$.

Proof. By proposition (26), it follows immediately.

\section{Corollary 28.}

Suppose that $X$ be a faithful finitely generated multiplication F. M. of $M$. Then every proper F. subm. of $X$ is T-ABSO iff every proper F. ideal of $\mathrm{R}$ is T-ABSO.

Proof. $(\Leftarrow)$ By corollary (27), it follows immediately.

$(\Longrightarrow)$ Let $\hat{\mathrm{H}}$ be a proper $\mathrm{F}$. ideal of $\mathrm{R}$. Then $A=\hat{\mathrm{H}} X$ is a proper subm. of $X$. Since $A$ is T-ABSO F. subm., so that $\left(A:_{R} X\right)$ is T-ABSO $\mathrm{F}$. ideal by theorem (21). But $\mathrm{X}$ is a multiplication $\mathrm{F}$. M., hence $A=\left(A:_{R} X\right) X$ by [5]. Thus $\hat{\mathrm{H}} X=\left(A:_{R} X\right) X$. Since $X$ is a faithful finitely generated multiplication F. M., then $X_{v}$ is a faithful finitely generated multiplication M. by $[16,17]$, implies that $X_{v}=\dot{M}$ is cancellation R-M. by [18]. Hence $X$ is a cancellation F. M. by [8]. Therefore $\hat{\mathrm{H}}=\left(A:_{R} X\right)$; that is $\hat{\mathrm{H}}$ is T-ABSO F. ideal of $\mathrm{R}$.

Recall that Let $X$ be F. M. of an R-M. $M$, and let $A$ be F. subm. of $X$. $A$ is called a pure F. subm., if for each $\mathrm{F}$. ideal $\hat{\mathrm{H}}$ of $\mathrm{R}$ such that $\hat{\mathrm{H}} A=\hat{\mathrm{H}} X \cap A$, see [19].

\section{Proposition 29.}

Let $A$ be a proper pure F. subm. of F. M. $X$ of $M$. If $0_{1}$ is T-ABSO F. subm. of $X$, then $A$ is T-ABSO F. subm. of $X$.

Proof. Let $a_{s} b_{l} x_{v} \subseteq A$ where $a_{s}, b_{l}$ F. singletons of $\mathrm{R}$ and $x_{v} \subseteq X$.

Put $\hat{\mathrm{H}}=<a_{s} b_{l}>$, hence $a_{s} b_{l} x_{v} \subseteq \hat{\mathrm{H}} X \cap A$, but $\hat{\mathrm{H}} X \cap A=\hat{\mathrm{H}} A$. So $a_{s} b_{l} x_{v}=a_{s} b_{l} y_{h}$, for some F. singleton $y_{h} \subseteq A$, then $a_{s} b_{l}\left(x_{v}-y_{h}\right) \subseteq 0_{1}$, but $0_{1}$ is T-ABSO F. subm., hence $a_{s}\left(x_{v}-y_{h}\right) \subseteq 0_{1}$ or $b_{l}\left(x_{v}-y_{h}\right) \subseteq 0_{1}$ or $a_{s} b_{l} \subseteq F-\operatorname{annX} \subseteq\left(A:_{R} X\right)$.

So we have $a_{s} x_{v}=a_{s} y_{h} \subseteq A$ or $b_{l} x_{v}=b_{l} y_{h} \subseteq A$ or $a_{s} b_{l} \subseteq\left(A:_{R} X\right)$.

Therefore $A$ is T-ABSO F. subm. of $X$.

Now, we give the concept of a cancellative F. M. as follows:

Definition 30. A F. M. $X$ of $\dot{M}$ is called a cancellative F. if whenever $a_{s} x_{v}=a_{s} y_{k}$ for F. singletons $a_{s}$ of $\mathrm{R}$ and $x_{v}, y_{k} \subseteq X, \forall s, v, k \in \mathrm{L}$, then $x_{v}=y_{k}$

\section{Proposition 31.}

Let $X$ be a cancellative F. M. of $M$, and A be a proper F. subm. of $X$. Then $A$ is a pure F. subm. of $X$ iff $A$ is T-ABSO F. subm.of $X$ with $\left(A:_{R} X\right)=0_{1}$. 
Proof. $(\Rightarrow)$ Assume that $A$ is a pure F. subm. of $X$ and $a_{s} b_{l} x_{v} \subseteq A$ such that $a_{s} b_{l} \nsubseteq$ $\left(A:_{R} X\right)$ for $\mathrm{F}$. singletons $a_{s}, b_{l}$ of $\mathrm{R}$ and $x_{v} \subseteq X$. Then $a_{s} b_{l} x_{v} \subseteq a_{s} b_{l} X \cap A=a_{s} b_{l} A$, hence $a_{s} b_{l} x_{v}=a_{s} b_{l} y_{k}$ for some F. singleton $y_{k} \subseteq A$. Since $X$ is a cancellative F. M., then $b_{l} x_{v}=$ $b_{l} y_{k} \subseteq A$. Thus $A$ is T-ABSO F. subm. of $X$.

Now, assume that F. singleton $r_{h} \subseteq\left(A:_{R} X\right)$ with $r_{h} \neq 0_{1}$. Since $A \neq X$ there exists F. singleton $x_{v} \subseteq X \backslash A$ such that $r_{h} x_{v} \subseteq r_{h} X \cap A=r_{h} A$, so there exists $\mathrm{F}$. singleton $y_{k} \subseteq A$, such that $r_{h} x_{v}=r_{h} y_{k}$, hence $x_{v}=y_{k}$ this is a contradication. So that $\left(A:_{R} X\right)=0_{1}$.

$(\Leftrightarrow)$ Suppose that $A$ is T-ABSO F. subm. of $X$. Let $a_{s} b_{l} x_{v} \subseteq a_{s} b_{l} X \cap A$ for F. singletons $a_{s}, b_{l}$ of $\mathrm{R}$ and $x_{v} \subseteq X$. We may suppose that $a_{s} b_{l} \neq 0_{1}$. Since $A$ is T-ABSO F. subm. of $X$, then either $a_{s} x_{v} \subseteq A$ or $b_{l} x_{v} \subseteq A$. If $b_{l} x_{v} \subseteq A$ and $b_{l}$ be F. singleton of $\mathrm{R}, a_{s} b_{l} x_{v} \subseteq a_{s} b_{l} A$. Thus $a_{s} b_{l} X \cap A \subseteq a_{s} b_{l} A$. By the same method to prove the case if $a_{s} x_{v} \subseteq A$; that is $a_{s} b_{l} A \subseteq$ $a_{s} b_{l} X \cap A$. Thus $a_{s} b_{l} X \cap A=a_{s} b_{l} A$. So that $A$ is a pure F. subm.

\section{T-ABSO Quasi Primary F. Subm.}

In this section we present the concept of T-ABSO quasi primary F. subm. and study the relationships this concept among T-ABSO F. subm. and T-ABSO primary F. subm. Many basic properties and outcomes are given. Now, we give the following definition:

\section{Definition 32.}

Let $A$ be a proper F. subm. of non-empty F. M. $X$ of an R-M. $M$. Then the $X$-F. radical of $A$, denoted by $X-\mathrm{R}(A)$ is defined to the intersection of all prime $\mathrm{F}$. subm. including $A$. We give the pursue lemma which are needed in the next proposition.

\section{Lemma 33.}

Let $X$ be a multiplication F. M. of $M$, let $A$ be a proper F. subm. of $X$. Then the following expressions are equivalent:

1- A is a prime F. subm. of $X$.

2- $\left(A:_{R} X\right)$ be a prime $\mathrm{F}$. ideal of $\mathrm{R}$.

3- $A=\hat{\mathrm{H}} X$ for some a prime $\mathrm{F}$. ideal $\hat{\mathrm{H}}$ of $\mathrm{R}$ with $F$-ann $X \subseteq \hat{\mathrm{H}}$.

Proof. (1) $\rightarrow$ (2) It follows by [20, proposition (2.5)].

$(2) \rightarrow(3)$ Since $X$ is a multiplication F. M., so that $A=\left(A:_{R} X\right) X$ by[5].

Put $\hat{\mathrm{H}}=\left(A:_{R} X\right)$ be a prime $\mathrm{F}$. ideal of $\mathrm{R}$. Now, since $F$-ann $X=\left(0_{1}:_{R} X\right)$ and $\left(0_{1}:_{R} X\right) \subseteq$ $\left(A:_{R} X\right)=\hat{\mathrm{H}}$. So that $F$-ann $X \subseteq \hat{\mathrm{H}}$.

(3) $\rightarrow$ (1) Let $a_{s} x_{v} \subseteq A$ for F. singleton $a_{s}$ of $\mathrm{R}$ and $x_{v} \subseteq X$, and $x_{v} \nsubseteq A$ to prove $a_{s} \subseteq\left(A:_{R} X\right)$. $\mathrm{By}(3), A=\hat{\mathrm{H}} X$ for some a prime $\mathrm{F}$. ideal $\hat{\mathrm{H}}$ of $\mathrm{R}$ with $F$-ann $X \subseteq \hat{\mathrm{H}}$, so that $F$-ann $X$ is a prime $\mathrm{F}$. ideal of R, but $F$-ann $X=\left(0_{1}:_{R} X\right)$, hence $\left(0_{1}:{ }_{R} X\right)$ is a prime $\mathrm{F}$. ideal of R. Let $a_{s} b_{l} \subseteq\left(0_{1}:_{R} X\right)$, for F. singleton $b_{l}$ of $\mathrm{R}$, and $b_{l} \nsubseteq\left(0_{1}:_{R} X\right)$, then $a_{s} \subseteq\left(0_{1}:_{R} X\right)$. Since $\left(0_{1}:_{R} X\right) \subseteq\left(A:_{R} X\right)$, so that $a_{s} \subseteq\left(A:_{R} X\right)$. Thus $A$ is a prime F. subm. of $X$.

\section{Lemma 34.}

Let $X$ be a finitely generated multiplication F. M. of $M$ and let $A$ be F. subm. of $X$. Then $X-R(A)=\sqrt{A:_{R} X} \cdot X$. 
Proof. If $X-\mathrm{R}(A)=X$, then the result is directly.

So that $X-\mathrm{R}(A) \neq X$, if $B$ is any prime $\mathrm{F}$. subm. of $X$ which contains $A$, we get $\left(A:_{R} X\right) \subseteq$ $\left(B:_{R} X\right)$. We prove that $\left(B:_{R} X\right)$ is a prime F. ideal. Assume that $a_{s} b_{l} \subseteq\left(B:_{R} X\right)$ for $\mathrm{F}$. singleton $a_{s}, b_{l}$ of $\mathrm{R}$, so that $a_{s} b_{l} X \subseteq B$, then either $b_{l} X \subseteq B$ or $b_{l} x_{v} \subseteq X / B$ for some $\mathrm{F}$. singleton $x_{v} \subseteq X$. But $B$ is a prime $\mathrm{F}$. subm. and $a_{s}\left(b_{l} x_{v}\right) \subseteq B$, then either $\left(b_{l} x_{v}\right) \subseteq B$ or $a_{s} \subseteq\left(B:_{R} X\right)$. Thus $a_{s} \subseteq\left(B:_{R} X\right)$ or $b_{l} \subseteq\left(B:_{R} X\right)$. So that $\left(B:_{R} X\right)$ is a prime F. ideal. Hence $\sqrt{A:_{R} X} \subseteq\left(B:_{R} X\right)$ by [13], then $\sqrt{A:_{R} X} . X \subseteq\left(B:_{R} X\right) X$. Since $B$ is an arbitary prime F. subm. containing $A$, we get $\sqrt{A:_{R} X} \cdot X \subseteq X-R(A)$

Now, since $X$ is a multiplication F. M., hence $X-R(A)=\left(X-R(A):_{R} X\right) X$.

We must prove that $\left(X-R(A):_{R} X\right) \subseteq \sqrt{A:_{R} X}$. Let $K$ be any prime F. ideal such that $\left(A:_{R} X\right) \subset K$. Since $K$ is a prime F. ideal containing $F$-ann $X=\left(0_{1}:_{R} X\right)$, then $K X$ is a prime F. subm. of $X$ containing $A=\left(A:_{R} X\right) X$ by lemma (33). Thus $\left(X-R(A):_{R} X\right) X=X-$ $R(A) \subseteq K X$, hence $\left(X-R(A):_{R} X\right) \subseteq K$, then $\left(X-R(A):_{R} X\right) \subseteq \sqrt{A:_{R} X}$ by [13], hence $X-R(A)=\left(X-R(A):_{R} X\right) X \subseteq \sqrt{A:_{R} X} . X$. So that $-R(A) \subseteq \sqrt{A:_{R} X} . X$

From (1) and (2), we get $-R(A)=\sqrt{A:_{R} X} \cdot X$.

Before the next proposition we give these lemmas and definition which are needed in the proof of the next proposition. We give this definition as follows:

\section{Definition 35.}

Let $X$ be F. M. of an R-M. $M$. If $\mathrm{P}$ is a maximal F. ideal of $\mathrm{R}$ then we define $F-G_{\mathrm{P}}(X)=$ $\left\{x_{v} \subseteq X:\left(1_{v}-a_{s}\right) x_{v}=0_{1}\right.$ for some F. sigleton $\left.a_{s} \subseteq \mathrm{P}, \forall v, s \in L\right\}$.

It is obvious $F-G_{\mathrm{P}}(X)$ is $\mathrm{F}$. subm. of $\mathrm{X} . \mathrm{X}$ is calld P-cyclic F. M. if there exist $\mathrm{F}$. singleton $b_{l} \subseteq \mathrm{P}$ and $x_{v} \subseteq X$ such that $\left(1_{v}-b_{l}\right) X \subseteq<x_{v}>, \forall l, v \in L$.

\section{Lemma 36.}

Let $\mathrm{R}$ be a commutative ring with unity. Then F. M. $X$ of an R-M. $M$ is a multiplication F. M. iff for every maximal F. ideal $\mathrm{P}$ of $\mathrm{R}$ either $X=F-G_{\mathrm{P}}(X)$ or $X$ is P-cyclic F. M.

Proof. $(\Rightarrow)$ Assume that $X$ is a multiplication F. M. Let $\mathrm{P}$ be maximal F. ideal of $\mathrm{R}$. Suppose that $X=\mathrm{P} X$, let $\mathrm{F}$. singleton $x_{v} \subseteq X$, then $\left\langle x_{v}\right\rangle=\hat{\mathrm{H}} X$ for some $\mathrm{F}$. ideal $\hat{\mathrm{H}}$ of $\mathrm{R}$. Hence $<x_{v}>=\hat{\mathrm{H}} X=\hat{\mathrm{HP}} X=\mathrm{PH} X=\mathrm{P}<x_{v}>$, then $x_{v}=a_{s} x_{v}$ for some F. sigleton $a_{s} \subseteq \mathrm{P}$. Thus $\left(1_{v}-a_{s}\right) x_{v}=0_{1}$, so that $x_{v} \subseteq F-G_{\mathrm{P}}(X)$. It follows that $X=F-$ $G_{\mathrm{P}}(X)$

Now, suppose that $X \neq \mathrm{P} X$, then there exists F. sigleton $x_{v} \subseteq X, x_{v} \nsubseteq \mathrm{P} X$. So that there exists an ideal $\mathrm{U}_{\Lambda}$ of $\mathrm{R}$ such that $\left\langle x_{v}\right\rangle=\mathrm{U}_{\Lambda} X$. It is obvious that $\mathrm{U}_{\Lambda} \nsubseteq \mathrm{P}$ and so $\left(1_{v}-b_{l}\right) \subseteq \mathrm{U}$ for some F. singleton $b_{l} \subseteq \mathrm{P}$. Hence $\left(1_{v}-b_{l}\right) X \subseteq<x_{v}>$. Thus $X$ is P-cyclic F. M. $(\Leftarrow)$ Suppose that for each maximal $\mathrm{F}$. ideal $\mathrm{P}$ of $\mathrm{R}$ either $X=F-G_{\mathrm{P}}(X)$ or $X$ is P-cyclic $\mathrm{F}$. M. Let $A$ be $\mathrm{F}$. subm. of $X$ and $\hat{\mathrm{H}}=\left(A:_{R} X\right)$. It is obvious that $\hat{\mathrm{H}} X \subseteq A$. Suppose that $\mathrm{F}$. singleton $y_{k} \subseteq A$ and $K=\left\{r_{h} \subseteq R: r_{h} y_{k} \subseteq \hat{\mathrm{H}} X\right\}$. Assume that $K \neq \mathrm{R}$, then there exists a maximal $\mathrm{F}$. ideal $E$ of $\mathrm{R}$ such that $K \subseteq E$ by [13, proposition(1.3.2.4)]. If $X=F-G_{E}(X)$ then $\left(1_{v}-a_{s}\right) y_{k}=$ $0_{1}$ for some F. singleton $a_{s} \subseteq E$, and $\left(1_{v}-a_{s}\right) \subseteq K \subseteq E$ this is a discrepancy. Thus by 
hypothesis there exist $\mathrm{F}$. singletons $b_{l} \subseteq E, z_{n} \subseteq X$ such that $\left(1_{v}-b_{l}\right) X \subseteq<z_{n}>$. It follows that $\left(1_{v}-b_{l}\right) A$ is $\mathrm{F}$. subm. of $\left\langle z_{n}\right\rangle$ and so tha $\left(1_{v}-b_{l}\right) A=D z_{n}$ where $D$ is $\mathrm{F}$. ideal $\left\{r_{h} \subseteq R: r_{h} z_{n} \subseteq\left(1_{v}-b_{l}\right) A\right\}$ of R. Note that $\left(1_{v}-b_{l}\right) D X=D\left(1_{v}-b_{l}\right) X \subseteq D z_{n} \subseteq A$. So that $\left(1_{v}-b_{l}\right) D \subseteq \hat{\mathrm{H}}$. Thus for $\mathrm{F}$. singleton $y_{k} \subseteq A,\left(1_{v}-b_{l}\right)^{2} y_{k} \subseteq\left(1_{v}-b_{l}\right)^{2} A=$ $\left(1_{v}-b_{l}\right) D z_{n} \subseteq \hat{\mathrm{H}} X$.

So that $\left(1_{v}-b_{l}\right)^{2} \subseteq K \subseteq E$ this is a discrepancy. Thus $K=\mathrm{R}$ and $y_{k} \subseteq \hat{\mathrm{H}} X$. Therefore $A=\hat{\mathrm{H}} X$ and $X$ is a multiplication F. M.

\section{Lemma 37.}

Let $X$ be a multiplication F. M. of an R-M. $M$, then $\cap_{i \in \Lambda}\left(\hat{\mathrm{H}}_{i} X\right)=\left(\cap_{i \in \Lambda}\left(\hat{\mathrm{H}}_{i}+F-a n n X\right)\right) X$ for any non-empty collection of F. ideals $\hat{\mathrm{H}}_{i}(i \in \Lambda)$ of R.

Proof. Assume that $X$ is a multiplication F. M. Let $\hat{\mathrm{H}}_{i}(i \in \Lambda)$ be any non-empty collection of F. ideals of $\mathrm{R}$, let $\mathrm{U}=\cap_{i \in \Lambda}\left(\hat{\mathrm{H}}_{i}+F-\operatorname{ann} X\right)$, then $\mathrm{U} X=\left(\cap_{i \in \Lambda}\left(\hat{\mathrm{H}}_{i}+F-\operatorname{ann} X\right)\right) X$. It is obvious that $\mathrm{U} X \subseteq \cap_{i \in \Lambda}\left(\hat{\mathrm{H}}_{i} X\right)$. Now, let be F. singleton $x_{v} \subseteq \cap_{i \in \Lambda}\left(\hat{\mathrm{H}}_{i} X\right)$ and let $G=$ $\left\{a_{s} \subseteq R: a_{s} x_{v} \subseteq \mathrm{U} X\right\}, \forall s, v \in L$ Suppose that $G \neq \mathrm{R}$, then there exists a maximal F. ideal $\mathrm{P}$ of $\mathrm{R}$ such that $G \subseteq \mathrm{P}$, it is obvious that $x_{v} \nsubseteq F-G_{\mathrm{P}}(X)$ and hence $X$ is P-cyclic F. M. by lemma (36). Then there exist F. singletons $a_{s} \subseteq \mathrm{P}$ and $y_{k} \subseteq X$ such that $\left(1_{v}-a_{s}\right) X \subseteq<y_{k}>$. Hence $\left(1_{v}-a_{s}\right) x_{v} \subseteq \bigcap_{i \in \Lambda}\left(\hat{\mathrm{H}}_{i} y_{k}\right)$. for each $i \in \Lambda$ there exists F. singleton $b_{l_{i}} \subseteq \hat{\mathrm{H}}_{i}, \forall l_{i} \in L$, such that $\left(1_{v}-a_{s}\right) x_{v}=b_{l_{i}} y_{k}$. Choose $j \in \Lambda$, for each $i \in \Lambda, b_{l_{j}} y_{k}=b_{l_{i}} y_{k}$, so that $\left(b_{l_{j}}-\right.$ $\left.b_{l_{i}}\right) y_{k}=0_{1}$, implies that: $\left(1_{v}-a_{s}\right)\left(b_{l_{j}}-b_{l_{i}}\right) X=\left(b_{l_{j}}-b_{l_{i}}\right)\left(1_{v}-a_{s}\right) X \subseteq\left(b_{l_{j}}-b_{l_{i}}\right)<y_{k}>$ $=0_{1},\left(1_{v}-a_{s}\right)\left(b_{l_{j}}-b_{l_{i}}\right)=0_{1}$. Thus $\left(1_{v}-a_{s}\right) b_{l_{j}}=\left(1_{v}-a_{s}\right) b_{l_{i}} \subseteq \hat{\mathrm{H}}_{i}(i \in \Lambda)$, then $\left(1_{v}-\right.$ $\left.a_{s}\right) b_{l_{j}} \subseteq \mathrm{U}_{\Lambda}$. Hence $\left(1_{v}-a_{s}\right)^{2} x_{v}=\left(1_{v}-a_{s}\right) b_{l_{j}} y_{k} \subseteq \mathrm{U}_{\Lambda} X$.

It follows that $\left(1_{v}-a_{s}\right)^{2} \subseteq G \subseteq \mathrm{P}$ this is a discrepancy. Thus $G=\mathrm{R}$ and $x_{v} \subseteq \mathrm{U} X$, so that $\cap_{i \in \Lambda}\left(\hat{\mathrm{H}}_{i} X\right) \subseteq \mathrm{U} X$ implies that $\mathrm{\cap}_{i \in \Lambda}\left(\hat{\mathrm{H}}_{i} X\right)=\mathrm{U} X$ That is $\mathrm{\cap}_{i \in \Lambda}\left(\hat{\mathrm{H}}_{i} X\right)=\left(\cap_{i \in \Lambda}\left(\hat{\mathrm{H}}_{i}+F-\right.\right.$ $\operatorname{ann} X)) X$. Now, we give the proposition as follows:

\section{Proposition 38.}

Let $X$ be a multiplication finitely generated F. M. of an R-M. $M$ and $A$ be T-ABSO F. subm. of $X$. Then one of the following satisfy:

$1-X-\mathrm{R}(A)=\mathrm{P}$ is a prime $\mathrm{F}$. subm. of $X$ such that $\mathrm{P}^{2} \subseteq A$.

2- $X-\mathrm{R}(A)=\mathrm{P}_{1} \cap \mathrm{P}_{2}, \mathrm{P}_{1} \mathrm{P}_{2} \subseteq A$ and $(X-R(A))^{2} \subseteq A$ where $\mathrm{P}_{1}, \mathrm{P}_{2}$ are the only distinct minimal prime $\mathrm{F}$. subms. of $A$.

Proof. By theorem (21), $\left(A:_{R} X\right)$ is T-ABSO F. ideal of R. So that either $R\left(\left(A:_{R} X\right)\right)=\mathrm{U}$ is a prime F. ideal of $\mathrm{R}$ such that $\mathrm{U}^{2} \subseteq\left(A:_{R} X\right)$ or $R\left(\left(A:_{R} X\right)\right)=\mathrm{U}_{1} \cap \mathrm{U}_{2}, \mathrm{U}_{1} \mathrm{U}_{2} \subseteq\left(A:_{R} X\right)$ and $R\left(\left(A:_{R} X\right)\right)^{2} \subseteq\left(A:_{R} X\right)$ where $\mathrm{U}_{1}, \mathrm{U}_{2}$ are the only distinct minimal prime $\mathrm{F}$. ideals of $\left(A:_{R} X\right)$ by proposition (6), where $R\left(\left(A:_{R} X\right)\right)=\sqrt{A:_{R} X}$. if the first case satisfies, then since $X$ is F. multiplication, we have $X-\mathrm{R}(A)=\mathrm{R}\left(\left(A:_{R} X\right)\right) X=\mathrm{U} X$ is a prime F. subm. of $X$. Put $\mathrm{U} X=\mathrm{P}$ by lemma (33) and lemma (34), and $(\mathrm{U} X)^{2}=\mathrm{U}^{2} X \subseteq\left(A:_{R} X\right) X=A$. Now, suppose that the latter case satisfies, then by lemma(33), $\mathrm{U}_{1} X$ and $\mathrm{U}_{2} X$ are the only distinct minimal prime $\mathrm{F}$. subms. 
of $A$ and $X-\mathrm{R}(A)=R\left(\left(A:_{R} X\right)\right) X=\left(\mathrm{U}_{1} \cap \mathrm{U}_{2}\right) X=\mathrm{U}_{1} X \cap \mathrm{U}_{2} X$ by lemma (37). Moreover $\left(\mathrm{U}_{1} X\right)\left(\mathrm{U}_{2} X\right)=\left(\mathrm{U}_{1} \mathrm{U}_{2}\right) X \subseteq\left(A:_{R} X\right) X=A \quad$ and $\quad(X-R(A))^{2}=\left(\mathrm{R}\left(\left(A:_{R} X\right)\right) \mathrm{X}\right)^{2}=$ $\left(\mathrm{R}\left(\left(A:_{R} X\right)\right)\right)^{2} X \subseteq\left(A:_{R} X\right) X=A$.

We give the definition of T-ABSO primary F. subm. as follows:

Definition 39. Let $A$ be a proper F. subm. of F. M. $X$ of $\dot{M}, A$ is called T-ABSO primary F. subm. of $X$ if whenever F. singletons $a_{s}, b_{l}$ of $\mathrm{R}$ and $x_{v} \subseteq X$ such that $a_{s} b_{l} x_{v} \subseteq A$, then either $a_{s} x_{v} \subseteq X-\mathrm{R}(A)$ or $b_{l} x_{v} \subseteq X-\mathrm{R}(A)$ or $a_{s} b_{l} \subseteq\left(A:_{R} X\right)$.

The following proposition characterize T-ABSO primary F. subm. in terms of its level subm.

\section{Proposition 40.}

Let $A$ be T-ABSO primary F. subm. of F. M. $X$ of Ḿ. for all $v \in \mathrm{L}$, iff the level subm. $A_{v}$ is T-ABSO primary subm. of $X_{v}$.

Proof. $(\Rightarrow)$ Let $a b x \in A_{v}$ for any $a, b \in \mathrm{R}$ and $x \subseteq X_{v}$, then $A(a b x) \geq v$, so $(a b x)_{v} \subseteq A$ implies that $a_{s} b_{l} x_{k} \subseteq A$ where $v=\min \{s, l, k\}$. Since $A$ be T-ABSO primary F. subm., so either $a_{s} x_{k} \subseteq X-R(A)$ or $b_{l} x_{k} \subseteq X-R(A)$ or $a_{s} b_{l} \subseteq\left(A:_{R} X\right)$.

If $a_{s} x_{k} \subseteq X-R(A)$, then $(a x)_{v} \subseteq X-R(A)$, so $a x \in X_{v}-R\left(A_{v}\right)$.

If $b_{l} x_{k} \subseteq X-R(A)$, then $(b x)_{v} \subseteq X-R(A)$, so $b x \in X_{v}-R\left(A_{v}\right)$.

If $a_{s} b_{l} \subseteq\left(A:_{R} X\right)$ then $(a b)_{v} \subseteq\left(A:_{R} X\right)$, so $a b \in\left(A:_{R} X\right)_{v}=\left(A_{v}:_{R} X X_{v}\right)$.

Hence $a b \in\left(A_{v}:_{R} X_{v}\right)$. Thus $A_{v}$ is T-ABSO primary subm. of $X_{v}$.

$(\Longleftarrow)$ Let $a_{s} b_{l} x_{k} \subseteq A$ for F. singletons $a_{s}, b_{l}$ of $\mathrm{R}$ and $x_{k} \subseteq X, \forall s, l, k \in \mathrm{L}$, hence $(a b x)_{v} \subseteq A$ where $v=\min \{s, l, k\}$ so that $A(a b x) \geq v$, implies $a b x \in A_{v}$, but $A_{v}$ is $\mathrm{T}$ ABSO primary subm. of $X_{v}$ so either $a x \in X_{v}-\mathrm{R}\left(A_{v}\right)$ or $b x \in X_{v}-\mathrm{R}\left(A_{v}\right) \quad$ or $a b \in\left(A_{v}:_{R} X_{v}\right)$. Since $\left(A_{v}:_{R} X_{v}\right)=\left(A:_{R} X\right)_{v}$, hence $a b \in\left(A:_{R} X\right)_{v}$. Then either $(a x)_{v} \subseteq$ $X-R(A)$ or $(b x)_{v} \subseteq X-\mathrm{R}(A)$ or $(a b)_{v} \subseteq\left(A:_{R} X\right)$, implies either $a_{s} x_{k} \subseteq X-R(A)$ or $b_{l} x_{k} \subseteq X-R(A)$ or $a_{s} b_{l} \subseteq\left(A:_{R} X\right)$. Thus $A$ be T-ABSO primary F. subm. of $X$.

\section{Remark 41.}

Every T-ABSO F. subm. is T-ABSO primary F. subm., but the converse in general incorrect, for example:

Let $X: Z \rightarrow L$ such that $X(y)=\left\{\begin{array}{cc}1 & \text { if } y \in Z \\ 0 & o . w .\end{array}\right.$

It is obvious that $X$ is F. M. of $Z$-M. $Z$.

Let $A: Z \rightarrow L$ such that $A(y)=\left\{\begin{array}{cc}v & \text { if } y \in 12 Z \\ 0 & \text { o.w. }\end{array} \forall v \in L\right.$

It is obvious that $A$ is $\mathrm{F}$. subm. of $X$.

Now, $A_{v}=12 Z$ and $X_{v}=Z$ as Z-M. Note that $A_{v}=12 Z$ is not T-ABSO subm. since 2.2.3 $\in 12 Z=A_{v}$ but $2.2 \notin 12 Z=A_{v}$ and $2.3 \notin 12 Z=A_{v}$.

But $X_{v}-\mathrm{R}\left(A_{v}\right)=Z-\mathrm{R}(12 Z)=2 Z \cap 3 Z=6 Z$ where $2 Z$ and $3 Z$ are prime subms. of $X_{v}$ containing $A_{v}$. So that $A_{v}$ is T-ABSO primary subm. of $X_{v}$ since $2.3=6 \in 6 Z$. Thus $A$ is not TABSO F. subm., but it is T-ABSO primary F. subm. of $X$. We give the concept of T-ABSO quasi primary F. subm. as follows: 
Definition 42. A proper F. subm. $A$ of F. M. $X$ of $M$ is called T-ABSO quasi primary F. subm. If $a_{s} b_{l} x_{v} \subseteq A$ implies either $a_{s} b_{l} \subseteq \sqrt{A:_{R} X}$ or $a_{s} x_{v} \subseteq X-R(A)$ or $b_{l} x_{v} \subseteq X-R(A)$ for each F. singleton $a_{s}, b_{l}$ of $\mathrm{R}$ and $x_{v} \subseteq X, \forall s, l, v \in \mathrm{L}$.

The following proposition characterize T-ABSO quasi primary $\mathrm{F}$. subm. in terms of its level subm.

\section{Proposition 43.}

Let $A$ be T-ABSO quasi primary F. subm. of F. M. $X$ of $M$ iff the level subm. $A_{v}$ is TABSO quasi primary subm. of $X_{v} \forall v \in \mathrm{L}$.

Proof. $(\Rightarrow)$ Let $a b x \in A_{v}$ for any $a, b \in \mathrm{R}$ and $x \in X_{v}$, then $A(a b x) \geq v$, so $(a b x)_{v} \subseteq A$ implies that $a_{s} b_{l} x_{k} \subseteq A$ where $v=\min \{s, l, k\}$. Since $A$ be a T-ABSO quasi primary F. subm., so either $a_{s} b_{l} \subseteq \sqrt{A:_{R} X}$ or $a_{s} x_{k} \subseteq X-R(A)$ or $b_{l} x_{k} \subseteq X-R(A)$.

If $a_{s} b_{l} \subseteq \sqrt{A:_{R} X}$ then $(a b)_{v} \subseteq \sqrt{A:_{R} X}$, so $a b$ $\in\left(\sqrt{A:_{R} X}\right)_{v}=\sqrt{A_{v}:_{R} X_{v}}$. Henc e $a b \in \sqrt{A_{v}:_{R} X_{v}}$.

If $a_{s} x_{k} \subseteq X-\mathrm{R}(A)$, then $(a x)_{v} \subseteq X-R(A)$, so $a x \in X_{v}-R\left(A_{v}\right)$.

If $b_{l} x_{k} \subseteq X-\mathrm{R}(A)$, then $(b x)_{v} \subseteq X-R(A)$, so $b x \in X_{v}-R\left(A_{v}\right)$.

Thus $A_{v}$ is a T-ABSO quasi primary subm. of $X_{v}$.

$(\Leftarrow)$ Let $a_{s} b_{l} x_{k} \subseteq A$ for $\mathrm{F}$. singletons $a_{s}, b_{l}$ of $\mathrm{R}$ and $x_{k} \subseteq X$, hence $(a b x)_{v} \subseteq A$ where $v=\min \{s, l, k\}$ so that $A(a b x) \geq v$, implies $a b x \in A_{v}$, but $A_{v}$ is T-ABSO quasi primary subm. of $X_{v}$, so either $a b \in \sqrt{A_{v}:_{R} X_{v}}$ or $a x \in X_{v}-R\left(A_{v}\right)$ or $b x \in X_{v}-R\left(A_{v}\right)$. Since $\sqrt{A_{v}:_{R} X_{v}}=$ $\left(\sqrt{A:_{R} X}\right)_{v}$, hence $a b \in\left(\sqrt{A:_{R} X}\right)_{v}$. Then either $(a b)_{v} \subseteq \sqrt{A:_{R} X}$ or $(a x)_{v} \subseteq X-R(A)$ or $(b x)_{v} \subseteq X-\mathrm{R}(A)$, implies either $a_{s} b_{l} \subseteq \sqrt{A:_{R} X} a_{s} x_{k} \subseteq X-R(A)$ or $b_{l} x_{k} \subseteq X-R(A)$ where $v=\min \{s, l, k\}$. Thus $A$ be T-ABSO quasi primary F. subm. of $X$.

\section{Theorem 44.}

Let $A$ be a proper F. subm. of F. M. $X$ of $M$. Then the following expressions are equivalent:

1 - $A$ is T-ABSO quasi primary F. subm. of $X$;

2- For every F. singleton $a_{s}, b_{l}$ of $\mathrm{R}, \forall s,\left(A:_{X} a_{s}^{n} b_{l}^{n}\right)=X$ for some $n \in Z^{+}$or $\left(A:_{X} a_{s} b_{l}\right) \subseteq\left(X-R(A):_{X} a_{s}\right) \cup\left(X-R(A):_{X} b_{l}\right)$.

3- For every F. singleton $a_{s}, b_{l}$ of $\mathrm{R}, \forall s, l \in \mathrm{L},\left(A:_{X} a_{s}^{n} b_{l}^{n}\right)=X$ for some $n \in Z^{+}$or $\left(A:_{X} a_{S} b_{l}\right) \subseteq\left(X-R(A):_{X} a_{s}\right)$ or $\left(A:_{X} a_{s} b_{l}\right) \subseteq\left(X-R(A):_{X} b_{l}\right)$.

Proof. (1) $\rightarrow$ (2) Assume that $A$ is T-ABSO quasi primary F. subm. of $X$, let F. singleton $a_{s}, b_{l}$ of $\mathrm{R}$.

If $a_{s} b_{l} \subseteq \sqrt{A:_{R} X}$, then $\left(a_{s} b_{l}\right)^{n}=a_{s}^{n} b_{l}^{n} \subseteq\left(A:_{R} X\right)$ for some $n \in Z^{+}$, hence $\left(A:_{X} a_{s}^{n} b_{l}^{n}\right)=X$. Now, suppose that $a_{s} b_{l} \nsubseteq \sqrt{A:_{R} X}$. Let $x_{v} \subseteq\left(A:_{X} a_{s} b_{l}\right)$, then $a_{s} b_{l} x_{v} \subseteq A$. Since $A$ is T-ABSO quasi primary F. subm., then $a_{s} x_{v} \subseteq X-R(A)$ or $b_{l} x_{v} \subseteq X-R(A)$. So that $\left(A:_{X} a_{s} b_{l}\right) \subseteq$ $\left(X-R(A):_{X} a_{s}\right) \cup\left(X-R(A):_{X} b_{l}\right)$.

$(2) \rightarrow(3)$ By $(2)$, we have $\left(A:_{X} a_{s} b_{l}\right) \subseteq\left(X-R(A):_{X} a_{s}\right) \cup\left(X-R(A):_{X} b_{l}\right)$.

So that $\left(A:_{X} a_{s} b_{l}\right) \subseteq\left(X-R(A):_{X} a_{s}\right)$ or $\left(A:_{X} a_{s} b_{l}\right) \subseteq\left(X-R(A):_{X} b_{l}\right)$. 
(3) $\rightarrow$ (1) Let $a_{s} b_{l} x_{v} \subseteq A$ and $a_{s} b_{l} \nsubseteq \sqrt{A:_{R} X}$ for F. singletons $a_{s}, b_{l}$ of $\mathrm{R}$ and $x_{v} \subseteq X$, hence $\left(a_{s} b_{l}\right)^{n}=a_{s}^{n} b_{l}^{n} \nsubseteq\left(A:_{R} X\right)$ for some $n \in Z^{+}$, then $\left(A:_{X} a_{s}^{n} b_{l}^{n}\right) \neq X$. By (3), we have that $x_{v} \subseteq$ $\left(A:_{X} a_{s} b_{l}\right) \subseteq\left(X-R(A):_{X} a_{s}\right)$ or $x_{v} \subseteq\left(A:_{X} a_{s} b_{l}\right) \subseteq\left(X-R(A):_{X} b_{l}\right)$. Thus $x_{v} a_{s} \subseteq X-$ $R(A)$ or $x_{v} b_{l} \subseteq X-R(A)$. So that $A$ is T-ABSO quasi primary F. subm. of $X$.

\section{Lemma 45.}

Let $X$ be F. M. of $M$. Suppose that $A$ is T-ABSO quasi primary F. subm. of $X$ and $a_{s} b_{l} B \subseteq$ $A$ for F. singleton $a_{s}, b_{l}$ of $\mathrm{R}, \forall s, l \in \mathrm{L}$, and F. subm. $B$ of $X$. If $a_{s} b_{l} \nsubseteq \sqrt{A:_{R} X}$, then $a_{s} B \subseteq$ $X-R(A)$ or $b_{l} B \subseteq X-R(A)$.

Proof. Since $B \subseteq\left(A:_{X} a_{s} b_{l}\right)$ and $\left(A:_{X} a_{s}^{n} b_{l}^{n}\right) \neq X$ for some $n \in Z^{+}$, by theorem (44), we get $B \subseteq\left(A:_{X} a_{s} b_{l}\right) \subseteq\left(X-R(A):_{X} a_{s}\right)$ or $B \subseteq\left(A:_{X} a_{s} b_{l}\right) \subseteq\left(X-R(A):_{X} b_{l}\right)$.

Then $a_{s} B \subseteq X-R(A)$ or $b_{l} B \subseteq X-R(A)$.

\section{Theorem 46.}

Let $A$ be a proper F. subm. of F. M. $X$ of $M$, then the following expressions are equivalent: $1-A$ is T-ABSO quasi primary F. subm. of $X$;

2- For F. singleton $a_{s}$ of $\mathrm{R}, \forall s \in \mathrm{L}, \mathrm{F}$. ideal $\hat{\mathrm{H}}$ of $\mathrm{R}$ and $\mathrm{F}$. subm. B of $\mathrm{X}$ with $a_{s} \hat{\mathrm{H}} B \subseteq A$, then either

$a_{S} \hat{\mathrm{H}} \subseteq \sqrt{A:_{R} X}$ or $a_{S} B \subseteq X-R(A)$ or $\hat{\mathrm{H}} B \subseteq X-R(A) ;$

3- For F. ideals $\hat{\mathrm{H}}$, $\mathrm{U}$ of $\mathrm{R}$, and F. subm. B of X with $\hat{\mathrm{H}} \mathrm{U} B \subseteq A$, then eithe $\hat{\mathrm{H} U} \subseteq \sqrt{A:_{R} X}$ or $\hat{\mathrm{H}} B \subseteq X-R(A)$ or $\mathrm{U} B \subseteq X-R(A)$.

Proof. (1) $\rightarrow$ (2) Assume that $a_{S} \hat{\mathrm{H}} B \subseteq A$ with $a_{S} \hat{\mathrm{H}} \nsubseteq \sqrt{A:_{R} X}$ and $\hat{\mathrm{H}} B \nsubseteq X-R(A)$. Then there exist $\mathrm{F}$. singletons $b_{l}, r_{k} \subseteq \hat{\mathrm{H}}$, such that $a_{s} b_{l} \nsubseteq \sqrt{A:_{R} X}$ and $r_{k} B \nsubseteq X-R(A)$. Now, we prove that $a_{s} B \subseteq X-R(A)$. Suppose that $a_{s} B \nsubseteq X-R(A)$. Since $a_{s} b_{l} B \subseteq A$, by lemma (45), we have $b_{l} B \subseteq X-R(A)$, hence $\left(b_{l}+r_{k}\right) B \nsubseteq X-R(A)$. By using lemma (45), we have $a_{s}\left(b_{l}+r_{k}\right)=a_{s} b_{l}+a_{s} r_{k} \subseteq \sqrt{A:_{R} X}$, because $a_{s}\left(b_{l}+r_{k}\right) B \subseteq A$. Since $a_{s} b_{l}+a_{s} r_{k} \subseteq$ $\sqrt{A:_{R} X}$ and $a_{s} b_{l} \nsubseteq \sqrt{A:_{R} X}$, we have $a_{s} r_{k} \nsubseteq \sqrt{A:_{R} X}$. Since $a_{s} r_{k} B \subseteq A$, by lemma (45), we have $r_{k} B \subseteq X-R(A)$ or $a_{s} B \subseteq X-R(A)$ this is a discrepancy. So that $a_{s} B \subseteq X-R(A)$.

$(2) \rightarrow$ (3) Suppose that $\hat{\mathrm{H} U} B \subseteq A$ with $\hat{\mathrm{H}} \mathrm{U} \Phi \nsubseteq \sqrt{A:_{R} X}$ for F. ideals $\hat{\mathrm{H}}$, U of R and F. subm. $B$ of $X$. Hence $a_{s} \mathrm{U} \nsubseteq \sqrt{A:_{R} X}$ for some F. singleton $a_{s} \subseteq \hat{\mathrm{H}}$. Now, we prove that $\hat{\mathrm{H}} B \subseteq X-R(A)$ or $\mathrm{U} B \subseteq X-R(A)$. Assume that $\hat{\mathrm{H}} B \nsubseteq X-R(A)$ and $\quad \mathrm{U} B \nsubseteq X-R(A)$. Since $a_{s} \mathrm{U} B \subseteq A$, by (2), we have $a_{s} B \subseteq X-R(A)$, then there exists $y_{h} \subseteq \hat{\mathrm{H}}$ such that $y_{h} B \nsubseteq X-R(A)$ since the assumption $\hat{\mathrm{H}} B \nsubseteq X-R(A)$. Since $y_{h} \mathrm{U} B \subseteq A$, we have $y_{h} \mathrm{U} \subseteq \sqrt{A:_{R} X}$, hence $\left(a_{S}+\right.$

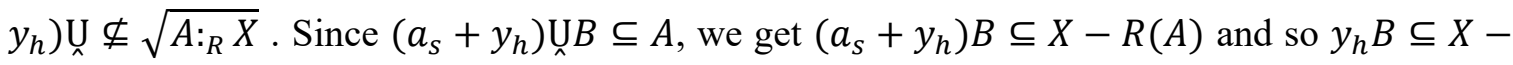
$R(A)$ this is a discrepancy. Thus $\hat{\mathrm{H}} B \subseteq X-R(A)$.

$(3) \rightarrow(1)$ Let $a_{s} b_{l} x_{v} \subseteq A$, for F. singletons $a_{s}, b_{l}$ of $\mathrm{R}$ and $x_{v} \subseteq X$. Put $\hat{\mathrm{H}}=<a_{s}>, \mathrm{U}=<$ $b_{l}>$ and $B=<x_{v}>$, then $\hat{\mathrm{H} U} \mathrm{U} B \subseteq A$. By (3), we have either $\hat{\mathrm{H} U} \cup \subseteq \sqrt{A:_{R} X}$ or $\hat{\mathrm{H}} B \subseteq X-$ $R(A)$ or $\mathrm{U} B \subseteq X-R(A)$; that is either $\left\langle a_{s}><b_{l}>\subseteq \sqrt{A:_{R} X}\right.$ or $<a_{s}><x_{v}>\subseteq X-$ 
$R(A)$ or $<b_{l}><x_{v}>\subseteq X-R(A)$. Hence either $a_{s} b_{l} \subseteq \sqrt{A:_{R} X}$ or $a_{s} x_{v} \subseteq X-R(A)$ or $b_{l} x_{v} \subseteq X-R(A)$. Thus $A$ is T-ABSO quasi primary F. subm. of $X$.

\section{Theorem 47.}

Let $X$ be F. M. of $M$, and $A$ be F. subm. of $X$. Then the following are satisfied:

1 - If is a multiplication F. M. and $\left(A:_{R} X\right)$ is T-ABSO quasi primary $\mathrm{F}$. ideal of $\mathrm{R}$, then $A$ is T- ABSO quasi primary F. subm. of $X$.

2- If $X$ is a finitely generated multiplication F. M. and $A$ is T-ABSO quasi primary F. subm. of $X$, then $\left(A:_{R} X\right)$ is T-ABSO quasi primary $\mathrm{F}$. ideal of $\mathrm{R}$.

Proof. (1) Assume that $X$ is a multiplication F. M., $\left(A:_{R} X\right)$ is T-ABSO quasi primary F. ideal of $\mathrm{R}$ and $\hat{\mathrm{H} U} U B \subseteq A$ for $\mathrm{F}$. ideals $\hat{\mathrm{H}}, \mathrm{U}$ of $\mathrm{R}$ and $\mathrm{F}$. subm. $B$ of $X$. Since $X$ is a multiplication $\mathrm{F}$.

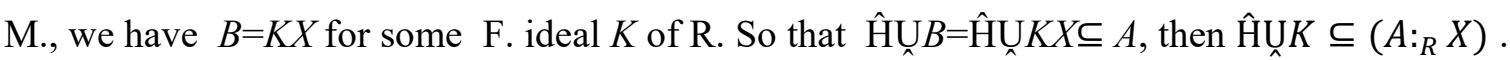
Since $\left(A:_{R} X\right)$ is T-ABSO quasi primary $\mathrm{F}$. ideal of $\mathrm{R}$, so by theorem (13), we have $\hat{\mathrm{H}} \mathrm{\Lambda} \subseteq$ $\sqrt{A:_{R} X}$ or $\hat{\mathrm{H}} K \subseteq \sqrt{A:_{R} X} \subseteq\left(X-R(A):_{R} X\right)$ or $\quad \mathrm{U} K \subseteq \sqrt{A:_{R} X} \subseteq\left(X-R(A):_{R} X\right)$. Hence $\hat{\mathrm{H} U} \subseteq \sqrt{A:_{R} X}$ or $\hat{\mathrm{H}} B \subseteq X-R(A)$ or $\mathrm{U} B \subseteq X-R(A)$. Then $A$ is T-ABSO quasi primary F. subm. of $X$ by theorem (46).

(2) Assume that $A$ is T-ABSO quasi primary $\mathrm{F}$. subm. of a finitely generated multiplication $\mathrm{F}$. M. $X$. Let F. singletons $a_{s}, b_{l}, r_{k}$ of $\mathrm{R}$, such that $a_{s} b_{l} r_{k} \subseteq\left(A:_{R} X\right)$ with $a_{s} b_{l} \nsubseteq \sqrt{A:_{R} X}$. Hence $a_{s} b_{l}\left(r_{k} x_{v}\right) \subseteq A$ for evey $\mathrm{F}$. singleton $x_{v} \subseteq X$. Since $A$ is T-ABSO quasi primary F. subm. of $X$ and $a_{s} b_{l} \nsubseteq \sqrt{A:_{R} X}$. Then we have $a_{s} r_{k} x_{v} \subseteq X-R(A)$ or $b_{l} r_{k} x_{v} \subseteq X-R(A)$ for all $x_{v} \subseteq X$. Hence we have $\left(X-R(A):_{X} a_{s} r_{k}\right) \cup\left(X-R(A):_{X} b_{l} r_{k}\right)=X$, so that $(X-$ $\left.R(A):_{X} a_{s} r_{k}\right)=X \quad$ or $\quad\left(X-R(A):_{X} b_{l} r_{k}\right)=X$. Then we have $a_{s} r_{k} \subseteq\left(X-R(A):_{R} X\right)=$ $\sqrt{A:_{R} X}$ or $b_{l} r_{k} \subseteq\left(X-R(A):_{R} X\right)=\sqrt{A:_{R} X}$. Thus $\left(A:_{R} X\right)$ is T-ABSO quasi primary F. ideal of $\mathrm{R}$.

\section{Theorem 48.}

Let $X$ be a finitely generated multiplication F. M. of $M$. For any F. subm. $A$ of $X$, the following expressions are equivalent:

1 - $A$ is T-ABSO quasi primary $\mathrm{F}$. subm. of $X$;

2- $X$-R $(A)$ is T-ABSO F. subm. of $X$.

Proof. (1) $\rightarrow$ (2) Assume that $A$ is T-ABSO quasi primary F. subm. of $X$. By theorm (47) and proposition (6), then we have $\sqrt{A:_{R} X}=\mathrm{U}_{\Lambda}$ is a prime F. ideal of $\mathrm{R}$ or $\sqrt{A:_{R} X}=\mathrm{U}_{1} \cap \mathrm{U}_{2}$ where $\mathrm{U}_{1}, \mathrm{U}_{2}$ are distinct prime F. ideals minimal over $\left(A:_{R} X\right)$. If $\sqrt{A:_{R} X}=\mathrm{U}$, hence $X$ $\mathrm{R}(A)=\mathrm{U} X$ is a prime subm. by lemma (43), so that $X-\mathrm{R}(A)$ is T-ABSO F. subm. of $X$.

Now, if $\sqrt{A:_{R} X}=U_{1} \cap U_{n}$ where $U_{1}, U_{\lambda}$ are distinct prime F. ideals minimal over $\left(A:_{R} X\right)$, then we have $X-\mathrm{R}(A)=\left(\mathrm{U}_{1} \cap \mathrm{U}_{2}\right) X$. Since $F$ - annX=(0 $\left.0_{1}:_{R} X\right)$ and $\left(0_{1}:_{R} X\right) \subseteq\left(A:_{R} X\right)$ and $\mathrm{U}_{1}, \mathrm{U}_{2}$ are distinct prime F. ideals minimal over $\left(A:_{R} X\right)$. So that $F-\operatorname{ann} X \subseteq \mathrm{U}_{1}, \mathrm{U}_{2}$. Then $X-\mathrm{R}(A)=\left(\left(\mathrm{U}_{1}+F-\right.\right.$ ann $\left.\left.X\right) \cap\left(\mathrm{U}_{2}+F-\operatorname{ann} X\right)\right) X=\mathrm{U}_{1} X \cap \mathrm{U}_{2} X$ by lemma (47). 
Since $\mathrm{U}_{1} X, \mathrm{U}_{2} X$ are two distinct prime F. subms., so that $X$-R $(A)$ is T-ABSO F. subm. of $X$ by remarks and examples(16)part(1).

$(2) \rightarrow(1)$ Assume that $X-\mathrm{R}(A)$ is T-ABSO F. subm. of $X$. Let $a_{s} b_{l} x_{v} \subseteq A$, for $\mathrm{F}$. singletons $a_{s}, b_{l}$ of $\mathrm{R}$ and $x_{v} \subseteq X$. Since $A \subseteq X-\mathrm{R}(A)$, then $a_{s} b_{l} x_{v} \subseteq X-R(A)$. But $X-\mathrm{R}(A)$ is T-ABSO F. subm. of $\mathrm{X}$, so that $a_{s} b_{l} \subseteq\left(X-R(A):_{R} X\right)=\sqrt{A:_{R} X}$ or $a_{s} x_{v} \subseteq X-R(A)$ or $b_{l} x_{v} \subseteq$ $X-R(A)$. Thus $A$ is T-ABSO quasi primary F. subm. of $X$. By combining theorem (47) and theorem (48), we get the following corollary is beneficial to determine T-ABSO quasi primary F. subm. of a finitely generated multiplication F. M.

\section{Corollary 49.}

For any F. subm. $A$ of a finitely generated multiplication F. M. $X$ of $M$. Then the following expressions are equivalent:

$1-A$ is T-ABSO quasi primary $\mathrm{F}$. subm. of $X$;

2- $X$ - $\mathrm{R}(A)$ is T-ABSO F. subm. of $X$;

3- $X-\mathrm{R}(A)$ is T-ABSO primary F. subm. of $X$;

4- $X-\mathrm{R}(A)$ is T-ABSO quasi primary F. subm. of $X$;

5- $\sqrt{A:_{R} X}$ is T-ABSO F. ideal of $\mathrm{R}$;

6- $\sqrt{A:_{R} X}$ is T-ABSO primary $\mathrm{F}$. ideal of $\mathrm{R}$,

7- $\sqrt{A:_{R} X}$ is T-ABSO quasi primary $\mathrm{F}$. ideal of $\mathrm{R}$;

8- $\left(A:_{R} X\right)$ is T-ABSO quasi primary $\mathrm{F}$. ideal of $\mathrm{R}$.

\section{Conclusions}

Through our research we concluded to the concepts (prime and quasi-prime) F. subm. lead to the concept T-ABSO F. subm. we reached the concept T-ABSO F. subm.one of the most important conclusions is the theorem (20), and explan the relationship if A is T-ABSO F. subm. with $\left(A:_{R} X\right)$ is T-ABSO F. ideal under the class of a multiplication $\mathrm{F}$. M. in corollary (23). Also we concluded the relationship $X-R(A)$ with $\sqrt{A:_{R} X}$ under the class of a multiplication F. M. in lemma (45), and explan the relationships $A$ is T-ABSO quasi primary F. subm.with $\left(A:_{R} X\right)$ is T-ABSO quasi primary $\mathrm{F}$. ideal and $A$ is T-ABSO quasi primary $\mathrm{F}$. subm.with $X-R(A)$ is T-ABSO F. subm. under the class of a multiplication $\mathrm{F}$. M. as in theorem (47), and theorem (48).

\section{References}

1. Deniz, S.; Gürsel, Y.; Serkan, O.; Bayram, A. E.; Bijan, D. On 2-Absorbing Primary Fuzzy Ideals of Commutative Rings. Mathematical Problems in Engineering. 2017, 2017, 1-7.

2. Lu. C. P. Prime Submodules of Modules. Commutative Mathematics. 1981, 33, 61-69.

3. Rabi. H. J. Prime Fuzzy Submodules and Prime Fuzzy Modules. M.Sc. Thesis. University of Baghdad. 2001.

4. Abdul-Razak, M. H. Quasi-Prime Modules and Quasi-Prime Submodules. M.Sc. Thesis. College of Education Ibn Al-Haitham. University of Baghdad. 1999.

5. Hatam, Y. K. Fuzzy Quasi-Prime Modules and Fuzzy Quasi-Prime Submodules. M.Sc. Thesis. University of Baghdad. 2001. 
6. Darani, A.Y.; Soheilnia, F. 2-Absorbing and Weakly 2-Absorbing Submodules. Thai Journal of Mathematics. 2011, 9, 3, 577-584.

7. Khalaf, H. Y.; Hannon, W. H. Small and Classical T-ABSO Fuzzy Submodules. Global Journal of Pure and Applied Mathematics. 2018, 14, 3, 443-457.

8. McCasland, R. L.; Moore, M. E. On Radical of Submodules of Finitely Generated Modules. Canad. Math. Bull. 1986, 29, 1, 37-39.

9. Mostafanasab, H.; Yetkin, E.; Tekir, U.; Darani, A. Y. On 2- absorbing Primary Submodules of Modules over Commutative Rings. An. St. Univ. Ovidius Constanta. 2016, 24, 1, 335-351.

10. Rabi, H. J.; Hassan, K. H. On Quasi Prime Fuzzy Submodules and Quasi Primary Fuzzy Submodules. J.Thi-Qar Sci. 2008, 1, 1, 37-42.

11. Suat, K.; Rabia, N.U.; Unsal, T. On 2-Absorbing Quasi Primary Submodules. Faculty of ciences and Mathematics. University of Ni`s, Serbia. 2017. 2943-2950.

12. Malik, D. S.; Mordeson, J. N. Fuzzy Maximal, Radical, and Primary Ideals of a Ring. Information sciences. 1991, 53, 237-250.

13. Hussein, W. R. Some Results of Fuzzy Rings. M.Sc. Thesis. University of Baghdad. 1999.

14. Abdulrahman, A. H. 2-Absorbing Submodules (Modules) and Some of Their Generalizations. M.Sc. Thesis. University of Baghdad. 2015.

15. Robert, W. Foundations of Module and Ring Theory. Gordon and Breach Science Publishers, 1991.

16. Rashed, H. G. Fully Cancellation Fuzzy Modules and Some Generalizations. M.Sc. Thesis. University of Baghdad. 2017.

17. Hadi, I. M. A.; Hamil, M. A. Cancellation and Weakly Cancellation Fuzzy Modules. Journal of Basrah Reserchs (Sciences). 2011. 37, 4.

18. El-Bast, Z. A.; Smith, P. F. Multiplication Modules.Comm.In Algebra. 1988, 16, $755-$ 779. 19. Maysoun, A. H. F-Regular Fuzzy Modules. M.Sc. Thesis. University of Baghdad. 2002.

19. Abou-Draeb, A. T.; Al-Zreegawi, B.; Al-Duja, Y. Kh. Prime Fuzzy Submodules and Strongly Prime Fuzzy Submodules Over Near-ring. Journal of Babylon Univ. Pure and Applied Science. 2014, 22, 3, 984-996. 